\title{
Deformations of cones over hyperelliptic curves
}

\author{
Jan Stevens
}

The deformation theory of a two-dimensional singularity, which is isomorphic to an affine cone over a curve, is intimately linked with the (extrinsic) geometry of this curve. In recent times various authors have studied one-parameter deformations, partly under the guise of extensions of curves to surfaces (cf. the survey [Wahl 1989]). In this paper we consider the versal deformation of cones, in the simplest case: cones over hyperelliptic curves of high degree. In particular, we show that for degree $4 g+4$, the highest degree for which interesting deformations exist, the number of smoothing components is $2^{2 g+1}$ (the case $g=3$ is exceptional).

Let $X$ be the cone over a hyperelliptic curve $C$, embedded with a line bundle $L$. If $d:=\operatorname{deg} L \geq 2 g+3$, then $X$ has always infinitesimal deformations in negative degrees: $\operatorname{dim} T_{X}^{1}(-1)=2 g+2$ [Drewes 1989]. On the other hand, one knows that only conical deformations exist over reduced base spaces, if $\operatorname{deg} L>$ $4 g+4$ [Tendian 1992a]. This implies that all deformations in negative degree must be obstructed. If $S$ is a surface with $C$ as hyperplane section, then one can degenerate $S$ to the projective cone over $C$, or from another point of view, deform the projective cone over $C$ to $S$; Pinkham calls this construction 'sweeping out the cone' [Pinkham 1970]. Surfaces with hyperelliptic hyperplane sections were already classified by Castelnuovo, and the supernormal surfaces among them have degree $4 g+4$ [Castelnuovo 1890]. They are rational ruled surfaces, and such surfaces come in two deformation types; therefore there are at least two smoothing components. This observation was the starting point of the present paper. A computer computation of the versal deformation in negative degree with Macaulay [Bayer-Stillman] gave for an example with $g=2$ the number of 32 smoothing components.

As the versal deformation can be chosen $\mathbb{C}^{*}$-equivariant, it makes sense to restrict to the part of negative degree. We want to show that the base space $S^{-}$has $2^{2 g+1}$ one-dimensional components. First of all we have to exhibit this number of surfaces with $C$ as hyperplane section, such that the normal bundle of $C$ in the surface is $L$. The main point is that an elementary transformation on the ruled surface $S$ in a Weierstraß point of $C$ does not change the normal bundle of $C$. The composition of elementary transformations in all Weierstraß points gives an involution on $S$; we get $2^{2 g+2} / 2$ surfaces. This construction works for every hyperelliptic curve $C$ and every line bundle $L$ on $C$. We obtain $2^{2 g+1}$ smooth subspaces of dimension $3 g$ of the base of the versal deformation; by a 
result of [Tendian 1992a] this dimension is exactly the dimension of smoothing components. Therefore we have found $2^{2 g+1}$ irreducible components.

The next thing to determine is $T^{2}$, the space where the obstructions lie. For $T^{2}(\nu), \nu<-2$, we have a general vanishing result [Wahl 1987], and it is not difficult to find the dimension in case $\nu>-2$. We find the dimension of $T^{2}$ with the MAIN LEMMA of [Behnke-Christophersen 1991], which connects the number of generators of $T^{2}$ with the codimension of smoothing components in the base space of the versal deformation of a general hyperplane section. Therefore we compute the dimension of $T^{1}$ for the cone over $d$ points on a rational normal curve of degree $d-g-1$ in $\mathbb{P}^{d-g-1}$. We use explicit equations for the curve. We also need the equations of $X$ to show that the $\mathcal{O}_{X}$-module $T^{2}$ is annihilated by the maximal ideal. Altogether we obtain that $\operatorname{dim} T^{2}(-2)=d-2 g-3, \operatorname{dim} T^{2}(-1)=(g-2)(d-g-3)$, and the other $T^{2}(\nu)$ vanish, if $d>2 g+3$.

Actually, the equations for the cone over a hyperelliptic curve, or for its hyperplane sections, have a nice structure, which Miles Reid calls the rolling factors format [Reid 1989]. We give an interpretation of $T^{2}(-2)$ in terms of the rolling factors format, in (2.12). It is not difficult to compute the part of the versal deformation in negative degrees, once one has represented $T^{1}(-1)$ as perturbations of the equations. Unfortunately, this representation is given by complicated formula's, which we only computed in the simplest case, that $L$ is a multiple of the $g_{2}^{1}$. The resulting equations for the base space $S^{-}$are as complicated, and it is difficult to see if they define a complete intersection; for $d=4 g+4$ we have $2 g+1$ quadratic equations in $2 g+2$ variables. By determining explicitly the elements of $T^{1}$, induced by our $2^{2 g+1}$ surfaces, we show that we have a complete intersection, and this allows us finally to conclude that there are no other components (except when $g=3)$.

Powerful methods exist to compute $T^{1}$ for surface singularities, without using explicit equations. They are based on Schlessinger's description of $T^{1}$ [Schlessinger 1973]: suppose $\operatorname{depth}_{0} X \geq 2$ and write $U=X \backslash 0$, then $T_{X}^{1}=\operatorname{ker}\left\{H^{1}\left(U, \Theta_{U}\right) \rightarrow\right.$ $\left.H^{1}\left(U,\left.\Theta_{n}\right|_{X}\right)\right\}$. In the special case that $X$ is the cone over a projective variety $Y$, all sheaves are graded, and the graded parts can be computed on $Y$, see [Schlessinger 1973] and Mumford's 'footnote' to it [Mumford 1973]. We describe the situation in terms of the sheaf of differential operators of order $\leq 1$ [EGA IV]. In the case of cones over curves it is advantageous to dualise. Now the bundle of principal parts comes in, and with it Wahl's Gaussian map (cf. [Wahl 1989]). For cones over curves of high degree the computation of $T^{1}(-1)$ is the most difficult; much of the work on the Gaussian map is connected with this case, and specially with vanishing results. The most complete results on interesting deformations are obtained by Sonny Tendian [Tendian 1990].

With a trick, which basically is contained in [Mumford 1973], one sees that for non hyperelliptic curves, embedded with a non special line bundle $L$, the dimension of $T^{1}(-1)$ of the cone over $C$ is equal to $h^{0}\left(C, N_{K} \otimes L^{-1}\right)$, where $N_{K}$ is the normal bundle of $C$ in its canonical embedding. For low genus this gives quite precise information, because then the normal bundle $N_{K}$ is easy to describe. We take the opportunity to remark that the computations in [Stevens 1989] imply that for a general curve of genus $g \geq 3$ the Gaussian map $\Phi_{K, L}$ is surjective for all 
line bundles $L$ with $\operatorname{deg} L \geq 2 g+11$. In the hyperelliptic case a variant of the construction yields easily the dimension of $T^{1}(-1)$.

The paper [Looijenga-Wahl 1986] introduces a collection of smoothing data for surface singularities, which in many cases distinguish between smoothing components. In our case we determine a subset with $1+2^{2 g}$ elements, onto which the set of smoothing components is mapped surjectively, so these smoothing data do not suffice to distinguish all components. The computations are similar to the case of a simple elliptic singularity of degree $8=4 g+4$, where the number of smoothing components is really $1+4$.

The organisation of this paper is as follows: in Section 1 we discuss the description of $T^{1}$ for cones, and give precise results in the hyperelliptic case. We also give a formula for the graded parts of $T^{2}$. In Section 2 we describe the equations for hyperelliptic cones. We compute $T^{1}$ for the general hyperplane section, and deduce the dimension of $T^{2}$ from it. In the last Section we prove the results on smoothing components.

\section{Cones over curves}

(1.1) The basic reference for the deformation theory of cones is a paper by Schlessinger [Schlessinger 1973] and Mumford's 'footnote' to it [Mumford 1973]. We start with the description of $T_{X}^{1}$ for a singularity $(X, 0) \subset\left(\mathbb{C}^{n}, 0\right)$ :

$$
\left.0 \longrightarrow \Theta_{X} \longrightarrow \Theta_{n}\right|_{X} \longrightarrow N_{X} \longrightarrow T_{X}^{1} \longrightarrow 0
$$

Here $N_{X}=\operatorname{Hom}\left(I / I^{2}, \mathcal{O}_{X}\right)$ is the normal sheaf; the tangent sheaf of $X$ is also defined as a dual: $\Theta_{X}=\operatorname{Hom}\left(\Omega_{X}^{1}, \mathcal{O}_{X}\right)$.

If $Z \subset X$ is a closed subset, containing the singular locus Sing $X$ of $X$, and if $\operatorname{depth}_{Z} X \geq 2$, then $T_{X}^{1}=\operatorname{coker}\left\{H^{0}\left(U,\left.\Theta_{n}\right|_{X}\right) \rightarrow H^{0}\left(U, N_{U}\right)\right\}$, or $T_{X}^{1}=$ $\operatorname{ker}\left\{H^{1}\left(U, \Theta_{U}\right) \rightarrow H^{1}\left(U,\left.\Theta_{n}\right|_{X}\right)\right\}$, where $U=X \backslash Z$. In the special case that $X$ is the cone over a projective variety $Y$ we want to interpret these groups on $Y$. From now on we work in the algebraic category.

Let $Y$ be a smooth projective variety, and $L$ a very ample line bundle on $Y$. We set $V=H^{0}(Y, L)$. Let $\phi_{L}: Y \rightarrow \mathbb{P}\left(V^{*}\right)$ be the embedding of $Y$ with $L$. Let $X \subset V^{*}$ be the affine cone over $\phi_{L}(Y)$. We will identify $Y$ and $\phi_{L}(Y)$; we have $L=\mathcal{O}_{Y}(1)$. Suppose that $\phi_{L}(Y)$ is projectively normal, i.e. $X$ is normal. The smooth space $U:=X-0$ is a $\mathbb{C}^{*}$-bundle over $Y$. We denote with the same symbol $\pi$ the projections $\pi: U \rightarrow Y$ and $\pi: V^{*}-0 \rightarrow \mathbb{P}\left(V^{*}\right)$. If $\mathcal{F}$ is a sheaf on $X$ with a natural $\mathbb{C}^{*}$-action, then $\pi_{*} \mathcal{F}$ decomposes into direct sums of the eigenspaces for the various characters of $\mathbb{C}^{*}$. Let $\mathcal{F}_{Y}$ be the sheaf of $\mathbb{C}^{*}$ invariants. Then:

$$
H^{0}(X, \mathcal{F})=H^{0}(U, \mathcal{F})=\bigoplus_{\nu=-\infty}^{\nu=\infty} H^{0}\left(Y, \mathcal{F}_{Y}(\nu)\right)
$$

The last equality does not hold in the analytic context; in that case one has a filtration on $H^{0}(X, \mathcal{F})$, whose associated graded space is the direct sum as above, which would suffice for our purposes. 
We describe the sheafs in the exact sequence $(*)$. Actually, when explicitly writing down sections, it is more convenient to use homogeneous coordinates, i.e. to compute on $X$. We illustrate this with the tangent bundle of $\mathbb{P}^{n}$. One has the Euler sequence [Hartshorne 1977, II.8.20.1]:

$$
0 \longrightarrow \mathcal{O}_{\mathbb{P}^{n}} \longrightarrow \mathcal{O}_{\mathbb{P}^{n}}(1)^{n+1} \longrightarrow \Theta_{\mathbb{P}^{n}} \longrightarrow 0 \text {. }
$$

In homogeneous coordinates $\left(z_{0}, \ldots, z_{n}\right)$ elements of a basis of $H^{0}\left(\mathcal{O}_{\mathbb{P}^{n}}(1)^{n+1}\right)$ can be written as $z_{i} \frac{\partial}{\partial z_{j}}$, and the map from $\mathcal{O}_{\mathbb{P}^{n}}$ is given by $1 \mapsto \sum z_{i} \frac{\partial}{\partial z_{i}}$.

We recall the definition of the sheaf of principal parts [Kleiman 1977, IV.A, EGA IV.16.7]. Consider a scheme $Y$; let $\mathcal{J}$ be the ideal sheaf of the diagonal $\Delta$ in $Y \times Y$, and let $Y_{\Delta}^{(n)}$ be the n-th infinitesimal neighbourhood of $\Delta$. The canonical projections $p_{1}$ and $p_{2}$ of the product induce maps $p_{1}^{(n)}: Y_{\Delta}^{(n)} \rightarrow Y$ and $p_{2}^{(n)}: Y_{\Delta}^{(n)} \rightarrow Y$. We define for a sheaf $\mathcal{F}$ [EGA IV. 16.7.2]:

$$
\mathcal{P}_{Y}^{n}(\mathcal{F})=\left(p_{1}^{(n)}\right)_{*}\left(\left(p_{1}^{(n)}\right)^{*}(\mathcal{F})\right)
$$

We write $\mathcal{P}_{Y}^{n}$ for $\mathcal{P}_{Y}^{n}\left(\mathcal{O}_{Y}\right)$. One has $\mathcal{P}_{Y}^{n}(\mathcal{F})=\mathcal{P}_{Y}^{n} \otimes \mathcal{O}_{Y} \mathcal{F}$, where the tensor product is taken with the $\mathcal{O}_{Y}$-module structure, defined by $p_{2}$. Because the diagonal is a section of $Y \times Y$ for both $p_{1}$ and $p_{2}$, both morphisms define a homomorphism $\mathcal{O}_{Y} \rightarrow \mathcal{P}_{Y}^{n}$, and therefore an $\mathcal{O}_{Y}$-module structure. Except when explicitly stated, we always consider the $\mathcal{O}_{Y}$-module structure on $\mathcal{P}_{Y}^{n}$, induced by $p_{1}$, and write it as left multiplication. One denotes by $d^{n}$ the morphism $\mathcal{O}_{Y} \rightarrow \mathcal{P}_{Y}^{n}$, induced by $p_{2}$ [EGA IV.16.3.6]. For every $t \in \Gamma\left(U, \mathcal{O}_{Y}\right), U \subset Y$ open, $d^{n} t$ is the principal part of order $n$. In particular, $d t=d^{1} t-t \in \Gamma\left(U, \Omega_{Y}^{1}\right)$ is the differential of $t$. We have the exact sequence:

$$
0 \longrightarrow \Omega_{Y}^{1} \longrightarrow \mathcal{P}_{Y}^{1} \longrightarrow \mathcal{O}_{Y} \longrightarrow 0
$$

On $\mathbb{P}^{n}$ this sequence is the dual of the Euler sequence.

The sheaf $\mathcal{P}_{Y}^{n}(\mathcal{F})$ has also two $\mathcal{O}_{Y}$-module structures; it is convenient to write them on the left and the right. For $a \in \Gamma\left(U, \mathcal{O}_{Y}\right), b \in \Gamma\left(U, \mathcal{P}_{Y}^{n}\right)$ and $t \in \Gamma(U, \mathcal{F})$ one has [EGA IV.16.7.4]:

$$
a(b \otimes t)=(a b) \otimes t, \quad(b \otimes t) a=\left(b \cdot d^{n} a\right) \otimes t=b \otimes(a t)=\left(d^{n} a\right) \cdot(b \otimes t) .
$$

There is a map $d_{\mathcal{F}}^{n}: \mathcal{F} \rightarrow \mathcal{P}_{Y}^{n}(\mathcal{F})$ with $d_{\mathcal{F}}^{n}(t)=1 \otimes t$.

(1.2) Definition [EGA IV.16.8.1]. Let $\mathcal{F}$ and $\mathcal{G}$ be two $\mathcal{O}_{Y}$-modules. A homomorphism $D: \mathcal{F} \rightarrow \mathcal{G}$ is a differential operator of order $\leq n$ if there exists a homomorphism $u: \mathcal{P}_{Y}^{n}(\mathcal{F}) \rightarrow \mathcal{G}$ such that $D=u \circ d_{\mathcal{F}}^{n}$.

The differential operators form a group; by applying the construction on open sets we obtain a sheaf $\mathcal{D}$ iff $_{Y}^{n}(\mathcal{F}, \mathcal{G})$, which is isomorphic to $\mathcal{H}_{\text {om }} \mathcal{O}_{Y}\left(\mathcal{P}_{Y}^{n}(\mathcal{F}), \mathcal{G}\right)$

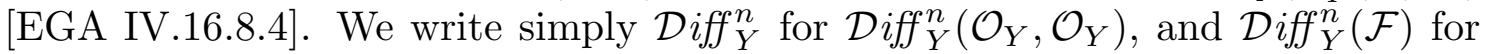
$\mathcal{D i f f}_{Y}^{n}\left(\mathcal{F}, \mathcal{O}_{Y}\right)$.

We return to our embedding $\phi_{L}: Y \rightarrow \mathbb{P}\left(V^{*}\right)$. Then $\phi_{L}^{*}\left(\mathcal{P}_{\mathbb{P}\left(V^{*}\right)}^{1}\right)=V \otimes_{\mathbb{C}} L^{-1}$. Here $V$ is considered to be the vector space with $d z_{i}$ as basis. Let $N_{Y}^{*}$ be the conormal bundle of $Y$ in $\mathbb{P}\left(V^{*}\right)$. Because $Y$ is smooth, one has the familiar exact sequence $0 \longrightarrow N_{Y}^{*} \longrightarrow \phi_{L}^{*}\left(\Omega_{\mathbb{P}\left(V^{*}\right)}^{1}\right) \longrightarrow \Omega_{Y}^{1} \longrightarrow 0$, with which we obtain the following result [Kleiman 1977, (IV.19)]: 
(1.3) Proposition. In the situation as above the following sequence is exact:

$$
0 \longrightarrow N_{Y}^{*} \otimes L \longrightarrow V \otimes_{\mathbb{C}} \mathcal{O}_{Y} \longrightarrow \mathcal{P}_{Y}^{1}(L) \longrightarrow 0,
$$

or dually:

$$
0 \longrightarrow \mathcal{D i f f}_{Y}^{1}(L) \longrightarrow V^{*} \otimes_{\mathbb{C}} \mathcal{O}_{Y} \longrightarrow N_{Y} \otimes L^{-1} \longrightarrow 0 .
$$

In particular we can view the $\mathbb{C}^{*}$-invariants of the exact sequence $(*)$ as obtained by taking global sections of the sequence $0 \longrightarrow \mathcal{D}_{\text {iff }}^{1} \longrightarrow V^{*} \otimes_{\mathbb{C}} L \longrightarrow$ $N_{Y} \longrightarrow 0$ on $Y$. We get the following formulation of a result of [Schlessinger 1973]:

(1.4) Theorem. Let $L$ be a very ample line bundle on a smooth projective variety $Y$, which embeds $Y$ as projectively normal subvariety of $\mathbb{P}\left(V^{*}\right)$, where $V=H^{0}(Y, L)$. Let $X \subset V^{*}$ be the affine cone over $Y$. Then the graded parts $T_{X}^{1}(\nu)$ of $T_{X}^{1}$ are given by:

$$
T_{X}^{1}(\nu)=\operatorname{coker}\left\{V^{*} \otimes H^{0}\left(Y, L^{\nu+1}\right) \longrightarrow H^{0}\left(Y, N_{Y} \otimes L^{\nu}\right)\right\},
$$

or alternatively,

$$
T_{X}^{1}(\nu)=\operatorname{ker}\left\{H^{1}\left(Y, \mathcal{D} i f f_{Y}^{1} \otimes L^{\nu}\right) \longrightarrow V^{*} \otimes H^{1}\left(Y, L^{\nu+1}\right)\right\} .
$$

From now one we concentrate on the case that $Y$ is one dimensional; Serre duality then transforms the second formula into one involving $H^{0}$.

(1.5) Corollary. Let $X$ be the cone over a curve $C$, embedded by $L=\mathcal{O}_{C}(1)$. Write $K$ for $\Omega_{C}^{1}$. Then:

$$
\left(T_{X}^{1}\right)^{*}(\nu)=\operatorname{coker}\left\{V \otimes H^{0}(C, K(-\nu-1)) \longrightarrow H^{0}\left(C, \mathcal{P}_{C}^{1} \otimes K(-\nu)\right)\right\} .
$$

This Corollary makes it possible to determine the graded parts of $T^{1}$ in many cases. Vanishing results exist for line bundles of high degree. We recall some results for the various degrees $\nu$. We assume that $g(C) \geq 2$.

(1.6) Case I: $\nu \geq 1$. Suppose first that $L^{\nu}$ is non special, i.e. $H^{1}\left(C, L^{\nu}\right)=0$. Then $T_{X}^{1}(\nu)=H^{1}\left(C, \mathcal{D} i f f_{C}^{1} \otimes L^{\nu}\right)=H^{1}\left(C, \Theta_{C} \otimes L^{\nu}\right)$. In particular, if $\operatorname{deg} L>4 g-4$, then $T_{X}^{1}(\nu)=0$ [Mumford 1973]. If $L^{\nu}$ is special, but $H^{1}\left(C, L^{\nu+1}\right)=0$, then still $T_{X}^{1}(\nu)=H^{1}\left(C, \mathcal{D}\right.$ iff $\left._{C}^{1} \otimes L^{\nu}\right)$. One has the exact sequence:

$$
0 \longrightarrow H^{1}\left(C, L^{\nu}\right) \longrightarrow H^{1}\left(C, \mathcal{D}_{i f f}^{1} \otimes L^{\nu}\right) \longrightarrow H^{1}\left(C, \Theta_{C} \otimes L^{\nu}\right) \longrightarrow 0 .
$$

If $L^{\nu}=K$, this follows from the surjectivity of $\Gamma\left(\mathcal{P}_{C}^{1}\right) \rightarrow \Gamma\left(\mathcal{O}_{C}\right)$.

(1.7) Case II: $\nu=0$. One has $T_{X}^{1}(0)=\operatorname{ker}\left\{H^{1}\left(C, \mathcal{D}\right.\right.$ iff $\left.\left.{ }_{C}^{1}\right) \longrightarrow V^{*} \otimes H^{1}(C, L)\right\}$. The map here can also be thought of as a cup product $H^{1}\left(C, \mathcal{D}\right.$ iff $\left.{ }_{C}^{1}\right) \otimes H^{0}(C, L) \longrightarrow$ $H^{1}(C, L)$ [Arbarello-Cornalba 1981]; for a differential operator $\theta$ and a section $s \in \Gamma(L)$ the cup product $\theta \cdot s=0 \in H^{1}(L)$ if and only if the section $s$ lifts to the first order deformation of $L \rightarrow C$, defined by $\theta$. In [loc.cit.] the vector space $T_{X}^{1}(0)$ is identified as tangent space to a space of $g_{d}^{r}$ s on a variable curve. More precisely, let $\pi: X \rightarrow S$ be a miniversal family of smooth curves, and consider the 
relative Picard variety $\operatorname{Pic}_{X / S}^{d}$ and the bundle $\mathcal{G}_{d}^{r}$ over $S$ of $g_{d}^{r}$ 's on the fibres; there is a map $c: \mathcal{W}_{d}^{r} \rightarrow \operatorname{Pic}_{X / S}^{d}$ with image $\mathcal{W}_{d}^{r}$. Because by assumption the linear series $L$ is complete, the map $c$ is injective. Then $H^{1}\left(C, \mathcal{D}\right.$ iff $\left.{ }_{C}^{1}\right)$ is the tangent space to $\operatorname{Pic}_{X / S}^{d}$ in the point $L \rightarrow C$, and $T_{X}^{1}(0)$ is the tangent space to $\mathcal{G}_{d}^{r}$. The problem now is to prove that $\mathcal{G}_{d}^{r}$ is smooth of expected dimension $3 g-3+\rho$. However, for $\rho<0$ not much is known.

For non special $L$ one has $\operatorname{dim} T_{X}^{1}(0)=4 g+3$. In terms of deformations this means that the cone can be deformed by changing the moduli of the curve or by changing the line bundle $L$ in $\operatorname{Pic}^{d}$. For $L=K$ the composed map $H^{1}\left(\mathcal{O}_{C}\right) \rightarrow$ $H^{1}\left(\mathcal{D}_{i f f}^{1}\right) \rightarrow V^{*} \otimes H^{1}(K) \cong H^{1}\left(\mathcal{O}_{C}\right)$ is an isomorphism, so $\operatorname{dim} T_{X}^{1}(0)=3 g-3$ and the versal family is a family of curves in their canonical embedding.

(1.8) Case III: $\nu \leq-2$. In this case $T_{X}^{1}(\nu)=H^{0}\left(C, N_{C} \otimes L^{\nu}\right)$ because $L^{\nu+1}$ is a line bundle of negative degree. We have two vanishing results.

(1.9) Lemma [Mumford 1973]. If $T_{X}^{1}(-1)=0$, then $T_{X}^{1}(\nu)=0$ for all $\nu \leq-2$.

Proof. If $\Gamma\left(N_{C} \otimes L^{\nu}\right) \neq 0$, then $N_{C} \otimes L^{-2}$ has a non-zero section $s$, and for all $t \in V=\Gamma(L)$ the tensor product $t \otimes s$ is a non-zero section of $N_{C} \otimes L^{-1}$; therefore $h^{0}\left(C, N_{C} \otimes L^{-1}\right) \geq \operatorname{dim} V$. Because $T_{X}^{1}(-1)=0$, the map $V^{*} \rightarrow \Gamma\left(N_{C} \otimes L^{-1}\right)$ is surjective, so all sections are of the form $t \otimes s$, therefore they are proportional, and do not generate $N_{C} \otimes L^{-1}$. But $N_{C} \otimes L^{-1}$ is generated by its sections, because $V^{*} \otimes \mathcal{O}_{C}$ is, and $V^{*} \otimes \mathcal{O}_{C} \rightarrow N_{C} \otimes L^{-1}$ is surjective.

(1.10) Remark. The argument in the proof shows that $\Gamma\left(N_{C} \otimes L^{\nu}\right)=0, \nu \leq-2$, if $\operatorname{dim} T_{X}^{1}(-1)<\operatorname{rank} N_{C}-1$.

(1.11) Proposition [Wahl 1987, 2.5]. Let $Y \subset \mathbb{P}=\mathbb{P}\left(V^{*}\right)$, with $V \subset \Gamma(L)$, be a projective variety, defined by a system of quadratic equations $\boldsymbol{f}$. Suppose every non-zero quadratic equation $f_{i}$ is involved in a linear relation $r$; this is true if the relations are generated by linear ones. Then $H^{0}\left(Y, N_{Y} \otimes L^{\nu}\right)=0$ for $\nu \leq-2$.

Proof. Let $\mathcal{I}$ be the ideal sheaf of $Y$. Consider the complex:

$$
\mathcal{O}_{\mathbb{P}}(-3)^{\oplus l} \stackrel{\boldsymbol{r}}{\longrightarrow} \mathcal{O}_{\mathbb{P}}(-2)^{\oplus k} \stackrel{f}{\longrightarrow} \mathcal{I} \longrightarrow 0,
$$

which is not necessarily exact at $\mathcal{O}_{\mathbb{P}}(-2)^{\oplus k}$. Dualise, twist and restrict to $Y$ to get:

$$
0 \longrightarrow N_{Y} \otimes L^{-2} \longrightarrow \mathcal{O}_{Y}^{\oplus k} \stackrel{{ }^{t} \boldsymbol{r}}{\longrightarrow} \mathcal{O}_{Y}(1)^{\oplus l} .
$$

Let $K$ be the kernel of the map ${ }^{t} \boldsymbol{r}: \mathbb{C}^{k} \rightarrow \Gamma(L)^{\oplus l}$. Here $\mathbb{C}^{k}$ can be identified with the dual of the vector space $Q$ of quadratic equations. The relations $\boldsymbol{r}$ involve only equations in $K^{\perp}$. Therefore $K=0$.

By a theorem of Green the conditions are satisfied for an embedding of a curve with a complete linear system of degree $d \geq 2 g+3$ [Green 1984, Thm. 4.a.1].

(1.12) Example. Let $C$ be hyperelliptic, with involution $\pi: C \rightarrow \mathbb{P}^{1}$, and let $L$ be very ample of degree $d$. Then $\phi_{L}(C)$ lies on a scroll $\bar{S}$, the image in $\mathbb{P}\left(\Gamma(C, L)^{*}\right)$ of $S=\mathbb{P}\left(\pi_{*} L\right)$, where $\pi_{*} L \cong \mathcal{O}(a) \oplus \mathcal{O}(b)$ with $a+b=d-(g+1)$, and $a, b \leq d / 2$. Suppose $b \leq a$, write $e=a-b$, so $0 \leq e \leq g+1$, then $S \cong \mathbb{P}(\mathcal{O} \oplus \mathcal{O}(-e))$. The 
Picard group of $S$ is generated by the section $E_{0}$ with $E_{0}^{2}=-e$, and the class $f$ of a fibre. We have $C \sim 2 E_{0}+(g+1+e) f$ (the coefficient of $f$ can be computed from the adjunction formula). Therefore $C^{2}=4 g+4$.

Now suppose that $d=\operatorname{deg} L=2 g+2$. Then $\bar{S}=S$, except when $b=0$, which occurs if $L=(g+1) g_{2}^{1}$; in that case $X$ is the cone over a rational normal curve of degree $g+1$, and $C$ does not pass through the vertex (because $E_{0} \cdot C=0$ ). We have the normal bundle exact sequence $\left.0 \rightarrow N_{C / S} \rightarrow N_{C} \rightarrow N_{\bar{S}}\right|_{C} \rightarrow 0$. Because the scroll $\bar{S}$ is defined by quadratic equations with linear relations, the argument of the proposition gives that $H^{0}\left(C, N_{\bar{S}} \otimes L^{-2}\right)=0$. Therefore $\Gamma\left(N_{C}(-2)\right)=$ $\Gamma\left(N_{C / S}(-2)\right)$. Because $L \sim E_{0}+a f$, we have $C \cdot(C-2 L)=C \cdot(g+1+e-2 a) f=0$, so $N_{C / S}(-2) \cong \mathcal{O}_{C}$ and $h^{0}\left(C, N_{C / S}(-2)\right)=1$. For $L=(g+1) g_{2}^{1}$ this result was obtained by Drewes [Drewes 1989].

We specialise to the case $g=3$. We proved in [Stevens 1991], that the cone $X$ over $\phi_{L}(C)$ has 3 smoothing components, if $L=2 K\left(=4 g_{2}^{1}\right)$, and 2 components otherwise. The curve $C$ is a complete intersection of the scroll and a quadric. If $L=2 K$, the scroll is the projective cone over a rational normal curve of degree 4 , which itself has two smoothing components; the deformation to the Veronese surface occurs, if we deform $C$ to a non hyperelliptic curve, with $L=2 \mathrm{~K}$.

We compute $T_{X}^{1}(-2)$ for $g(C)=3, \operatorname{deg} L=8, C$ not hyperelliptic. If $L=2 K$, we find as above that $\Gamma\left(N_{C}(-2)\right)=\Gamma\left(N_{C / S}(-2)\right)=\Gamma\left(\mathcal{O}_{C}\right)$, where $S$ is the Veronese surface. If $L \neq 2 K$, let $D$ be a general divisor in the linear system; it is cut out on the canonical curve $C_{4}$ in $\mathbb{P}^{2}$ by a cubic $C_{3}$, and the linear system is the system of cubics through the residual intersection $C_{4} \cap C_{3}-D$. Therefore $C \subset \mathbb{P}^{5}$ lies on (non unique) Del Pezzo surface of degree 5. One checks that all equations (which are quadratic) occur in linear relations, although the relations are not generated by linear ones. So $T_{X}^{1}(-2)=0$. A smoothing is obtained by sweeping out the cone over the blow-up of $\mathbb{P}^{2}$ with the linear system of quartics with base points in $D$. By [Tendian 1992a, Prop. 6.1] the dimension of the corresponding smoothing component is 15 , which is also the dimension of $T^{1}$ (see the description of $T^{1}(-1)$ below), so the base space is smooth. For $L=2 K$ this component has codimension 1, and there is a second component; if $C=S \cap\{Q=0\}$, then $S \cap\left\{Q=t^{2}\right\}$ is $\mathbb{P}^{2}$, branched in $C_{4}$.

(1.13) Case IV: $\nu=-1$. This is the most difficult case. A more specific knowledge of the maps in the theorem is necessary. We review the relation with Wahl's Gaussian map [Wahl 1990], see also [Tendian 1990, Drewes 1989, Wahl 1989].

We start with the following diagram:

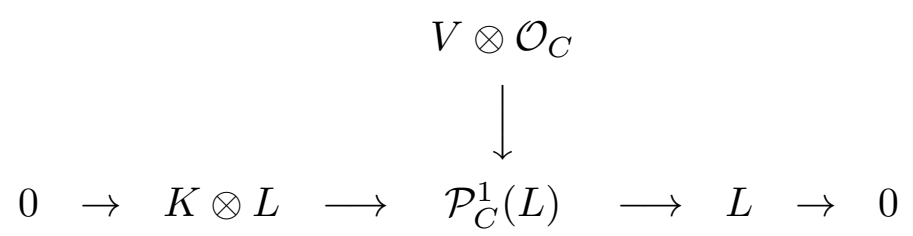

The kernel of the composed map $V \otimes \mathcal{O}_{C} \rightarrow L$ is a vector bundle $\mathcal{M}_{L}$ over $C$, and we get a map $\mathcal{M}_{L} \rightarrow K \otimes L$. Let $M$ be a second line bundle, and tensor everything with $M$. Define:

$$
\mathcal{R}(L, M)=\operatorname{ker}\left\{\mu_{L, M}: \Gamma(L) \otimes \Gamma(M) \rightarrow \Gamma(L \otimes M)\right\} .
$$


Then $\mathcal{R}(L, M)=\Gamma\left(\mathcal{M}_{L} \otimes M\right)$ and we have the Gaussian map $\Phi_{L, M}: \mathcal{R}(L, M) \rightarrow$ $\Gamma(K \otimes L \otimes M)$. This map is given explicitly by $\Phi_{L, M}(\alpha)=\sum d_{L}^{1} l_{i} \otimes m_{i}$, where $\alpha=$ $\sum l_{i} \otimes m_{i} \in \mathcal{R}(L, M)$ with $l_{i} \in \Gamma(L)$ and $m_{i} \in \Gamma(M)$. A more symmetric definition can be given in local coordinates; represent sections on an open set $U$ by functions, again denoted by $l_{i}$ and $m_{i}$. Then from $\sum l_{i} m_{i}=0$ we get $\sum\left(l_{i} d m_{i}+m_{i} d l_{i}\right)=0$, so $\Phi_{L, M}(\alpha)$ can be represented by the 1 -form $1 / 2 \sum\left(l_{i} d m_{i}-m_{i} d l_{i}\right)$.

For curves we have that $H^{1}(K \otimes L \otimes M)=0$, so if we denote the map $\Gamma(L) \otimes \Gamma(M) \rightarrow \Gamma\left(\mathcal{P}_{C}^{1}(L) \otimes M\right)$ by $d_{L}^{1} \otimes 1_{M}$, we have the exact sequence $0 \rightarrow$ coker $\Phi_{L, M} \rightarrow$ coker $d_{L}^{1} \otimes 1_{M} \rightarrow$ coker $\mu_{L, M} \rightarrow 0$.

We can also start the construction above with $M$ in stead of $L$. Up to a permutation of factors one has $\mu_{L, M}=\mu_{M, L}$ and $\Phi_{L, M}=-\Phi_{M, L}$. Therefore:

$$
\operatorname{coker} d_{L}^{1} \otimes 1_{M} \cong \operatorname{coker} d_{M}^{1} \otimes 1_{L} \text {. }
$$

We apply this to the computation of $T_{X}^{1}(-1)$. From Corollary (1.5) we have $\left(T_{X}^{1}(\nu)\right)^{*}=$ coker $d_{L}^{1} \otimes 1_{K \otimes L^{-(\nu+1)}}$ and in particular $\left(T_{X}^{1}(-1)\right)^{*}=\operatorname{coker} d_{L}^{1} \otimes 1_{K}$. Therefore $\left(T_{X}^{1}(-1)\right)^{*} \cong \operatorname{coker} d_{K}^{1} \otimes 1_{L}$.

If $\phi_{L}: C \rightarrow \mathbb{P}\left(H^{0}(C, L)^{*}\right)$ is birational onto its image, then the multiplication maps $\mu_{L, K \otimes L^{-(\nu+1)}}$ are surjective [Arbarello-Sernesi 1978, Proof of Thm 1.6]. This applies to our situation, so $\left(T_{X}^{1}(\nu)\right)^{*}=\operatorname{coker} \Phi_{L, K \otimes L^{-(\nu+1)}}$ and in particular $\left(T_{X}^{1}(-1)\right)^{*}=\operatorname{coker} \Phi_{L, K}=\operatorname{coker} \Phi_{K, L}$.

Now suppose that $C$ is not hyperelliptic. Then $K$ is very ample and we have the exact sequence:

$$
0 \longrightarrow N_{K}^{*} \otimes K \longrightarrow \Gamma(K) \otimes_{\mathbb{C}} \mathcal{O}_{C} \longrightarrow \mathcal{P}_{C}^{1}(K) \longrightarrow 0,
$$

where $N_{K}^{*}$ is the conormal bundle of the canonical embedding. This yields that $\left(T_{X}^{1}(-1)\right)^{*}=\operatorname{ker}\left\{H^{1}\left(N_{K}^{*} \otimes K \otimes L\right) \rightarrow \Gamma(K) \otimes H^{1}(L)\right\}$. This map is surjective, because $H^{1}\left(\mathcal{P}_{C}^{1}(K) \otimes L\right)=0$. Dually, $T_{X}^{1}(-1)=\operatorname{coker}\left\{\Gamma(K)^{*} \otimes H^{0}\left(K \otimes L^{-1}\right) \rightarrow\right.$ $\left.H^{0}\left(N_{K} \otimes L^{-1}\right)\right\}$. In particular:

(1.14) Lemma. For the cone $X$ over a non hyperelliptic curve, embedded by a non special complete linear system, $T_{X}^{1}(-1)=H^{0}\left(N_{K} \otimes L^{-1}\right)$.

As Mumford remarks, there is an integer $d_{0}$, depending only on $C$, such that $H^{0}\left(N_{K} \otimes L^{-1}\right)=0$ for $\operatorname{deg} L \geq d_{0}$. The bundle $\mathcal{E}$ of [Mumford 1973] is $N_{K} \otimes K^{-1}$; in fact, Mumford's construction can be understood as interchanching $K$ and $L$.

In general it is difficult to give sharp explicit bounds, but for low genus the result is very effective. Before we give some examples, we prove a result [Wahl $1989,2.2]$, which is based on a more general lemma of Lazarsfeld, giving sufficient conditions for surjectivity of Gaussian maps.

(1.15) Lemma. Suppose $C$ is not hyperelliptic, trigonal or a plane quintic. If $H^{0}\left(K^{2} \otimes L^{-1}\right)=0$ (in particular, if $\left.\operatorname{deg} L>4 g-4\right)$, then $\Phi_{K, L}$ is surjective.

Proof. By Petri's Theorem the ideal $I$ of the canonical curve $C$ is generated by quadrics, so there is a surjection $\mathcal{O}(-2)^{\otimes a} \rightarrow I$. Dualising, restricting to $C$ and twisting gives an injection $H^{0}\left(N_{K} \otimes L^{-1}\right) \rightarrow\left(H^{0}\left(K^{2} \otimes L^{-1}\right)\right)^{\otimes a}$.

We now look at low genera. 
(1.16) $g=3$. The normal bundle is the line bundle $K^{\otimes 4}$. Therefore $d_{0}=17$. For $\operatorname{deg} L=16$ only $L=K^{\otimes 4}$ has non zero $T^{1}(-1)$, and the cone $X\left(C, K^{\otimes 4}\right)$ is smoothable, as hyperplane section of $X\left(\mathbb{P}^{2}, \mathcal{O}(4)\right)$. For all $L$ with $\operatorname{deg} L=13$ the Gaussian map $\Phi_{K, L}$ is not surjective, for the general $L$ of degree 14 it is.

(1.17) $g=4$. The normal bundle is $K^{\otimes 2} \oplus K^{\otimes 3}$, so $d_{0}=19$.

More generally, for $C$ trigonal, the canonical curve sits on a scroll $S$ as divisor of type $3 H-(g-4) R$ [Schreyer 1986], and we have the normal bundle sequence $\left.0 \rightarrow N_{C / S} \rightarrow N_{C} \rightarrow N_{S}\right|_{C} \rightarrow 0$. Here $N_{C / S}=K^{3} \otimes\left(g_{3}^{1}\right)^{4-g}$, a bundle of degree $3 g+6$. Sonny Tendian proves that $H^{1}\left(\left.N_{S}\right|_{C} \otimes L^{-1}\right)=0$, if $H^{0}\left(K^{2} \otimes L^{-1}\right)=0$, or for $L=K^{2}$ [Tendian 1990]. In particular, $d_{0} \leq \max (3 g+7,4 g-4)$. We conjecture that $H^{1}\left(\left.N_{S}\right|_{C} \otimes L^{-1}\right)=0$ if $\operatorname{deg} L \geq d_{1}$ for some $d_{1}<3 g+3$. For $\operatorname{deg} L=3 g+6$ we would then have that $T^{1}(-1) \neq 0$ only if $L$ is the characteristic linear system of the family of curves of type $3 H-(g-4) R$ on $S$, which also gives a one parameter smoothing.

(1.18) $g=5$. The general curve is a complete intersection of three quadrics, with normal bundle $3 K^{2}$, and $d_{0}=17$ (for a trigonal ${ }^{5} C$ we have $d_{0}=22$ ).

(1.19) $g=6$. Here the famous possibility of a plane quintic occurs. It gives $d_{0}=26$. The general curve lies on a (possibly singular) Del Pezzo surface $S$ of degree 5 with normal bundle $N_{C / S}=K^{2}$.

Tendian has studied the case $L=K^{2}$ [Tendian 1990]. He proves that $\Phi_{K, K^{2}}$ is surjective, if the Clifford index of $C$ is at least 3. This leaves the hyperelliptic, trigonal and tetragonal curves, as well as smooth plane quintics and sextics. We now concentrate on the tetragonal case. Then $C$ lies on a three dimensional scroll $X$ of type $S\left(e_{1}, e_{2}, e_{3}\right)$ of degree $e_{1}+e-2+e_{3}=g-3$ [Schreyer 1986], but more important are the surfaces on which $C$ lies, cf. [Lévy-Bruhl-Mathieu 1953]. We obtain $C$ as complete intersection of divisors $\mathrm{Y} \sim 2 \mathrm{H}-b_{1} \mathrm{R}$ and $\mathrm{Z} \sim 2 \mathrm{H}-b_{2} \mathrm{R}$ on $X$ with $b_{1}+b_{2}=g-5$ and $b_{2} \leq b_{1}$. Either $Y$ is rational, or the given $g_{4}^{1}$ is composed with an elliptic or hyperelliptic involution $C \stackrel{2: 1}{\longrightarrow} E \stackrel{2: 1}{\longrightarrow} \mathbb{P}^{1}$, and $Y$ is a ruled surface over $E$, with a rational curve of double points, which is the canonical image of $E$; then $\operatorname{deg} Y=g-1+b_{2}, p_{a}(E)=1 / 2\left(b_{2}+2\right)$, and $C$ does not intersect the double curve. The normal bundle of $C$ in $Y$ is $N_{C / Y}=K^{2} \otimes\left(g_{4}^{1}\right)^{-b_{2}}$. In the case $b_{2}=0$, and $g>5, c$ lies on a unique elliptic cone $Y$, and $H^{1}\left(C, N_{Y} \otimes K^{-2}\right)=0$ [Tendian 1990, 2.2.12], so $\operatorname{dim} T_{X\left(C, K^{2}\right)}^{1}(-1)=1$ and $Y$ leads to a one parameter deformation to a simple elliptic singularity of degree $g-1$.

The examples above, where $\Phi_{K, L}$ is not surjective, have in common that $N_{K}$ is unstable; the rank of $N_{K}$ is $g-2$, the degree is $2\left(g^{2}-1\right)=(g-2)(2 g+4)+6$, and we found surfaces $Y$ with $\operatorname{deg} N_{C / Y}>2 g+4+\lfloor 6 / g-2\rfloor$. This motivates:

(1.20) Question. Suppose that the canonical curve ${ }^{g} C$ lies on a unique surface $Y$ with $d_{0}=\operatorname{deg} N_{C / Y}>2 g+4+\lfloor 6 / g-2\rfloor$. Is then $\Phi_{K, L}$ surjective for all $L$ with $\operatorname{deg} L>d_{0}$, and for $L$ with $\operatorname{deg} L=d_{0}$ and $L \neq N_{C / Y}$ ?

For a general curve such surfaces do not exist; already for $g=7$ the general curve has a plane representation as $C^{7}\left(8 A^{2}\right)$, so $\operatorname{deg} N_{C / Y}=17$.

(1.21) Question [Wahl 1989, 2.5]. Is $\Phi_{K, L}$ surjective on a general curve of genus $g \geq 12$ for $\operatorname{deg} L \geq 2 g-2$ ? What is the best bound? 
We remark that for general $C$ with $g=10$ or $g \geq 12$ the map $\Phi_{K, K}$ is surjective [Ciliberto-Harris-Miranda 1988]. The problem is of course to find a general curve; the easiest to handle are singular curves. In [Stevens 1989] we constructed for all $g \geq 5$ a $g$-cuspidal canonically embedded curve $\Gamma$ with a Weil divisor $D$ of degree $g+5$ such that $h^{0}\left(N_{\Gamma}(-D)\right)=6$.

(1.22) Proposition. For a general curve of genus $g \geq 3$ the map $\Phi_{K, L}$ is surjective for all $L$ with $\operatorname{deg} L \geq 2 g+11$.

Proof. From [Stevens 1989, Prop. 5] it follows that $h^{0}\left(N_{C} \otimes L^{-1}\right) \leq 6$ for a general curve $C$ with $g \leq 5$ and a general $L$ with $\operatorname{deg} L=g+5$. Therefore $h^{0}\left(N_{C} \otimes L^{-1}\right)=0$, if $\operatorname{deg} L \geq g+11$; presumably a general $L$ of degree $g+6$ will do for large $g$. Fix a line bundle $L_{0}$ of degree $g+11$ with $h^{0}\left(N_{C} \otimes L_{0}^{-1}\right)=0$. Every line bundle $L$ with $\operatorname{deg} L \geq 2 g+11$ can be written as $L=L_{0}(D)$ with $D$ an effective divisor of degree $\operatorname{deg} L-g-11 \geq q$. This implies that $H^{0}\left(N_{C} \otimes L^{-1}\right)$ is a subspace of $H^{0}\left(N_{C} \otimes L_{0}^{-1}\right)$. For $g=3,4$ see (1.16) and (1.17).

(1.23) The hyperelliptic case. The formula:

$$
\left(T_{X}^{1}(-1)\right)^{*}=\operatorname{coker} d_{L}^{1} \otimes 1_{K} \cong \operatorname{coker} d_{K}^{1} \otimes 1_{L}
$$

still holds. However the map $d_{K}^{1}: \Gamma(K) \otimes \mathcal{O}_{C} \rightarrow \mathcal{P}^{1}(K)$ is only generically surjective, with cokernel equal to coker $\left\{\phi_{K}^{*} \Omega_{\mathbb{P}}^{1} \otimes K \rightarrow K^{2}\right\} \cong \Omega_{\phi} \otimes K$, where $\Omega_{\phi}$ are the relative differentials, so the cokernel is given by the Jacobian ideal [Piene 1978]. Therefore, if $B$ denotes the set of Weierstraß points, we have an exact sequence:

$$
\Gamma(K) \otimes \mathcal{O}_{C} \stackrel{d_{K}^{1}}{\longrightarrow} \mathcal{P}^{1}(K) \longrightarrow \bigoplus_{p \in B} \mathbb{C}_{p} \rightarrow 0 .
$$

Let $\mathcal{P}=\operatorname{Im} d_{K}^{1}$; this is a rank 2 vector bundle.

(1.24) Lemma. Let the rational normal curve $R=\phi_{K}(C)$ be the canonical image of $C$, and write $\phi: C \rightarrow R$; let $H=\mathcal{O}_{R}(1) \cong \mathcal{O}_{\mathbb{P}^{1}}(g-1)$. Let $N_{R}$ be the normal bundle of $R$ in $\mathbb{P}^{g-1}$. The following sequence is exact:

$$
0 \rightarrow \phi^{*}\left(N_{R}^{*} \otimes H^{-1}\right) \longrightarrow \Gamma(K) \otimes \mathcal{O}_{C} \stackrel{d_{K}^{1}}{\longrightarrow} \mathcal{P} \rightarrow 0 .
$$

Proof. Consider the commutative diagram:

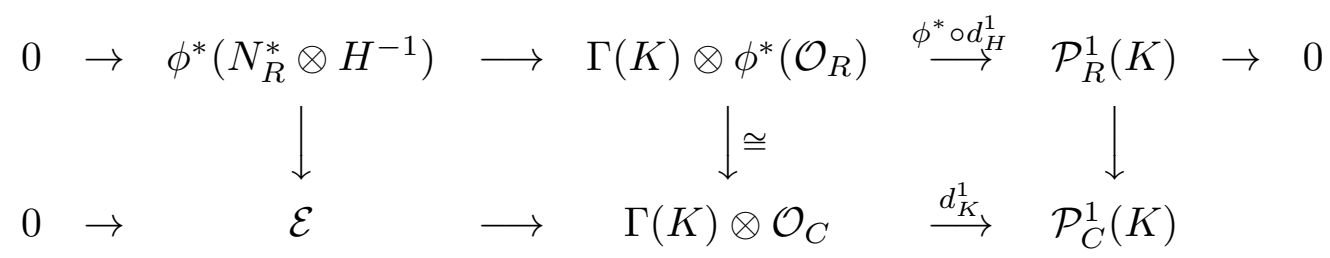

Because $\phi^{*} \circ d_{H}^{1}=d_{K}^{1} \circ \phi^{*}$ [EGA IV.16.4.3.4] the image of the right-hand vertical map is $\mathcal{P}$, and therefore $\mathcal{E}=\phi^{*}\left(N_{R}^{*} \otimes H^{-1}\right)$.

The normal bundle of $R$ splits a direct sum of $g-2$ bundles of degree $g+1$, and therefore $\mathcal{E}=\oplus_{g-2}\left(g_{2}^{1}\right)^{-2}$. Using the fact that $H^{1}\left(\mathcal{P}_{C}^{1}(K) \otimes L\right)=0$ for $\operatorname{deg} L>0$, we obtain the following result; for $\operatorname{deg} L>2 g+1$ cf. [Wahl 1988, 7.11.1, Drewes 1989]. 
(1.25) Proposition. Let $C$ be a hyperelliptic curve of genus $g$, and $L$ a line bundle with $\operatorname{deg} L>0$. Then dim coker $d_{K}^{1} \otimes 1_{L}=2 g+2+(g-2) h^{1}\left(L \otimes\left(g_{2}^{1}\right)^{-2}\right)-$ $g h^{1}(L)$. In particular, if $\operatorname{deg} L>2 g+2$, then $\operatorname{dim} T^{1}(-1)=2 g+2$.

We remark that $H^{1}(L)=0$, if $L$ defines a birational map of $C$. Then $h^{1}(L \otimes$ $\left.\left(g_{2}^{1}\right)^{-2}\right) \leq 1$, as one sees from the exact sequence $0 \rightarrow L \otimes\left(g_{2}^{1}\right)^{-2} \rightarrow L \rightarrow \mathbb{C}^{4} \rightarrow 0$; if $h^{0}\left(L \otimes\left(g_{2}^{1}\right)^{-2}\right)=0$, this follows because $h^{0}(L) \geq 3$; therefore we may assume that $H^{0}\left(L \otimes\left(g_{2}^{1}\right)^{-2}\right)=\left\{\psi, \ldots, \psi z^{k}\right\}$ for some function $\psi$ on $C$ and a local coordinate $z$ on $\mathbb{P}^{1}$. Then $H^{0}(L)$ contains in addition the section $\psi z^{i+1}, \psi z^{i+2}$ and at least one section which is not of this form.

(1.26) $T^{2}$. We recall the definition of $T_{X}^{2}$ for a singularity $(X, 0) \subset\left(\mathbb{C}^{n}, 0\right)$ [Schlessinger 1973]: let $0 \rightarrow R \rightarrow\left(\mathcal{O}_{n}\right)^{\oplus k} \stackrel{j}{\longrightarrow} I \rightarrow 0$ be a resolution of the ideal $I$ of $X$. Let $R_{0}$ be the submodule of $R$, generated by the trivial relations, i.e. those of the form $x j(y)-y j(x)$. Then $R / R_{0}$ is a $\mathcal{O}_{X}$-module, and $T_{X}^{2}=\operatorname{coker}\left\{\operatorname{Hom}\left(\left(\mathcal{O}_{X}\right)^{\oplus k}, \mathcal{O}_{X}\right) \rightarrow \operatorname{Hom}\left(R / R_{0}, \mathcal{O}_{X}\right)\right\}$. We can also consider the exact sequence $0 \rightarrow R_{X} \rightarrow\left(\mathcal{O}_{X}\right)^{\oplus k} \rightarrow I / I^{2} \rightarrow 0$ on $X$. There is a surjection $R / R_{0} \rightarrow R_{X}$, whose kernel is a torsion module for reduced $X$. Therefore we get the alternative description:

$$
0 \rightarrow N_{X} \longrightarrow \operatorname{Hom}\left(\left(\mathcal{O}_{X}\right)^{\oplus k}, \mathcal{O}_{X}\right) \longrightarrow \operatorname{Hom}\left(R_{X}, \mathcal{O}_{X}\right) \longrightarrow T_{X}^{2} \rightarrow 0
$$

Suppose the sheaf $\mathcal{T}^{2}$ has support contained in $Z$ with $\operatorname{dp}_{Z} X \geq 2$, and let $U=$ $X \backslash Z$. Then $T_{X}^{2}=\operatorname{coker}\left\{H^{0}\left(U, \mathcal{O}_{n}^{\oplus k}\right) \rightarrow H^{0}\left(U, R_{X}^{*}\right)\right\}=\operatorname{ker}\left\{H^{1}\left(U, N_{X}\right) \rightarrow\right.$ $\left.\left.H^{1}\left(U,\left(\mathcal{O}_{n}\right)^{\oplus k}\right)\right)\right\}$.

We specialise to the case of cones as before, so $X$ is the cone over the smooth projective variety $Y$, embedded with the line bundle $L$. Let the ideal of $Y$ in $\mathbb{P}\left(V^{*}\right), V=H^{0}(Y, L)$, be generated by $k$ equations of degree $d_{1}, \ldots, d_{k}$. Then the graded parts of $T_{X}^{2}$ are given by the exact sequence:

$$
0 \rightarrow T_{X}^{2}(\nu) \longrightarrow H^{1}\left(Y, N_{Y}(\nu)\right) \longrightarrow H^{1}\left(\mathcal{O}_{Y}\left(d_{1}+\nu\right)\right) \oplus \cdots \oplus H^{1}\left(\mathcal{O}_{Y}\left(d_{k}+\nu\right)\right) .
$$

If $\operatorname{dim} Y=1$, then the group $H^{1}\left(Y, N_{Y}(\nu)\right)$ occurs in the following exact sequence, where we write $C$ for $Y$ :

$$
0 \rightarrow T_{X}^{1}(\nu) \longrightarrow H^{1}\left(\mathcal{D} i f f_{C}^{1}(\nu)\right) \longrightarrow V \otimes H^{1}\left(C, L^{\nu+1}\right) \longrightarrow H^{1}\left(C, N_{C}(\nu)\right) \rightarrow 0 .
$$

This gives a formula for the dimension of $T_{X}^{2}(\nu)$ :

$\operatorname{dim} T_{X}^{2}(\nu)=\operatorname{dim} \operatorname{ker}\left\{V \otimes H^{1}\left(L^{\nu+1}\right) \rightarrow H^{1}\left(\oplus_{i} L^{d_{i}+\nu}\right)\right\}-h^{1}\left(\mathcal{D} i f f_{C}^{1}(\nu)\right)+\operatorname{dim} T_{X}^{1}(\nu)$.

If $H^{1}(C, L)=0$, then $H^{1}\left(C, N_{C}(\nu)\right)=0$ for $\nu \geq 0$, and therefore also $T_{X}^{2}(\nu)=0$. If $C$ is defined by quadratic equations, and $L$ is not special, then $T_{X}^{2}(-1)=$ $H^{1}\left(C, N_{C}(-1)\right)$ and therefore $\operatorname{dim} T_{X}^{2}(-1)=(g-2) h^{0}(L)-6(g-1)+\operatorname{dim} T_{X}^{1}(-1)$, because $h^{1}\left(\mathcal{D}\right.$ iff $\left.{ }_{C}^{1}(-1)\right)=2 \operatorname{deg} L+4 g-4$. 
(1.27) Example. The above computation gives new examples of singularities for which the obstruction map is not surjective, cf. [Tendian 1990, 2.4.1]. In particular, if $g(C)=3$ and $L$ is general of degree $d \geq 14$, then $\operatorname{dim} T_{X}^{1}(-1)=$ 0 , and $X$ has only conical deformations, so the base space is smooth, whereas $\operatorname{dim} T_{X}^{2}(-1)=d-14$. For $g=4$ and general $L$ with $9 \leq d=\operatorname{deg} L<15$ we have $\operatorname{dim} T_{X}^{1}(-1)=15-d, \operatorname{dim} T_{X}^{1}=28-d$, which is also the dimension of a smoothing component [Tendian 1992a, 6.2]. Therefore the base space is smooth, but $\operatorname{dim} T_{X}^{2}(-1)=d-9$. Finally, for a general $X\left({ }^{5} C, L_{d}\right)$ with $d>12$ there are only conical deformations, and $\operatorname{dim} T_{X}^{2}(-1)=3(d-12)$.

For $T_{X}^{2}(\nu)$ with $\nu<-2$ we have the following general vanishing result:

(1.28) Lemma [Wahl, Cor. 2.10]. If $Y \subset \mathbb{P}=\mathbb{P}^{n}$ is a smooth projectively normal subvariety, defined by quadratic equations, with a resolution

$$
\mathcal{O}_{\mathbb{P}}(-4)^{\oplus m} \longrightarrow \mathcal{O}_{\mathbb{P}}(-3)^{\oplus l} \longrightarrow \mathcal{O}_{\mathbb{P}}(-2)^{\oplus k} \longrightarrow \mathcal{O}_{\mathbb{P}} \longrightarrow \mathcal{O}_{X} \longrightarrow 0
$$

then the cone $X$ over $Y$ satisfies $T_{X}^{2}(\nu)=0$ for $\nu<-2$.

By [Green 1984, Thm. 4.a.1] the conditions are satisfied for a curve, embedded with a complete linear system of degree $d \geq 2 g+4$.

\section{The versal deformation of hyperelliptic cones}

(2.1) Our aim in this section is to compute equations of the versal deformation of the cone $X=X(C, L)$ over a hyperelliptic curve ${ }^{g} C$, embedded with a complete linear system $L$ of degree at least $2 g+3$. Then, as we have seen, $T_{X}^{1}$ is concentrated in degrees 1, 0 and -1 , and $T_{X}^{1}(1)=0$ if $\operatorname{deg} L>4 g-4$. We restrict ourselves to the part of the versal deformation in negative degree, because otherwise also non hyperelliptic curves come in.

For the computation of $T^{1}$ efficient methods exist, which avoid the explicit use of equations and relations. For the versal deformation there seems to be no alternative. Actually, as Frank Schreyer repeatedly pointed out to me, the equations for $\phi_{L}(C)$ are rather simple in the hyperelliptic case: $C$ is a divisor on a two-dimensional scroll, so besides the determinantal of the scroll we have 'essentially' one equation.

(2.2) Equations for $X$. Let $L$ be any line bundle of degree $d \geq 2 g+3$. Denote the involution by $\pi: C \rightarrow \mathbb{P}^{1}$. Then $\phi_{L}(C)$ lies on the scroll $S=\mathbb{P}_{\mathbb{P}^{1}}\left(\pi_{*} L\right)$, where $\pi_{*} L \cong \mathcal{O}(a) \oplus \mathcal{O}(b)$ with $a+b=d-(g+1)$, and $a, b \leq d / 2$; in particular, if $L=k g_{2}^{1}$, then $a=k$ and $b=k-(g+1)$. Suppose $b \leq a$, write $e=a-b$, so $0 \leq e \leq g+1$, and $S \cong \mathbb{P}(\mathcal{O} \oplus \mathcal{O}(-e))$. In Pic $S=\mathbb{Z} E_{0} \oplus \mathbb{Z} f$, where $E_{0}$ is the section with $E_{0}^{2}=-e$ and $f$ is the class of a fibre, we have $C \sim 2 E_{0}+(g+1+e) f$.

Let $L=k g_{2}^{1}$ with $k>g+1$. Let $C$ be the curve $y^{2}-\sum_{i=0}^{2 g+2} a_{i} x^{i}=0$. We can make this equation quasi-homogeneous by introducing homogeneous coordinates $(x, \bar{x})$ on $\mathbb{P}^{1}$, so $C=\left\{y^{2}-\sum_{i=0}^{2 g+2} a_{i} \bar{x}^{2 g+2-i} x^{i}=0\right\}$. A basis for $H^{0}(C, L)$ is given by the functions:

$$
z_{i}=\bar{x}^{k-i} x^{i}, \quad i=0, \ldots, k ; \quad w_{i}=y \bar{x}^{k-g-1-i} x^{i}, \quad i=0, \ldots, k-g-1 .
$$


The equations for $X$ are those for the scroll, and $2 k-2 g-1$ further equations $\phi_{m}$, obtained by multiplying the equation of $C$ by suitable powers of $\bar{x}$ and $x$, such that the result can be expressed in the $z_{i}$ and $w_{i}$. These equations are obtained from one another by rolling factors, as Miles Reid puts it [Reid 1989]. For each monomial in an equation we get the corresponding monomial in the next equation by multiplying with $x / \bar{x}$, that is, with the quotient of the entries in a suitable column of the matrix defining the scroll. We make here a specific choice; we recall the notation $\lceil r\rceil$ for the round up and $\lfloor r\rfloor$ for rounding off a real number $r$, and note that $\left\lceil\frac{m}{2}\right\rceil=\left\lfloor\frac{m+1}{2}\right\rfloor$ for integers $m$. This gives the following equations:

$$
\begin{gathered}
\operatorname{Rank}\left(\begin{array}{ccccccc}
z_{0} & z_{1} & \ldots & z_{k-1} & w_{0} & \ldots & w_{k-g-2} \\
z_{1} & z_{2} & \ldots & z_{k} & w_{1} & \ldots & w_{k-g-1}
\end{array}\right) \leq 1, \\
\phi_{m}=w_{\left\lfloor\frac{m}{2}\right\rfloor} w_{\left\lceil\frac{m}{2}\right\rceil}-\sum_{i=0}^{2 g+2} a_{i} z_{\left\lfloor\frac{m+i}{2}\right\rfloor^{\left.z \frac{m+i}{2}\right\rceil},}
\end{gathered}
$$

If $L$ is an arbitrary bundle of degree $d \geq 2 g+3$, then we can write $L=k g_{2}^{1}+D$ with $\operatorname{deg} D=g+1-e$, and $k$ maximal such that $D$ is effective. The divisor $D$ can be described by two polynomials $U(x)$ and $V(x)$, together with $F(x)=\sum_{i=0}^{2 g+2} a_{i} x^{i}$ [Mumford 1984, $\S 1$ ]. Suppose $D=P_{1}+\cdots+P_{g+1-e}$ with all $P_{i}$ distinct and let $\left(x_{i}, y_{i}\right)$ be the coordinates of $P_{i}$. Define $U(x)=\prod_{i}\left(x-x_{i}\right)$, and take $V(x)$ the unique polynomial of degree $\leq g-e$ with $V\left(x_{i}\right)=y_{i}$. The ideal $(U, y-V)$ defines $D$; this is indeed a subvariety of $y^{2}-F$, and one has $F-V^{2}=U W$ for some polynomial $W$.

The function $(y+V) / U$ defines a section of $H^{0}(C, L)$; a basis of this vector space can be represented in inhomogeneous coordinates by the polynomial forms:

$$
z_{i}=U x^{i}, \quad i=0, \ldots, k ; \quad w_{i}=(y+V) x^{i}, \quad i=0, \ldots, k-e .
$$

Modulo the equation $y^{2}-F$ we have the relation $(y+V)^{2}=U W+2(y+V) V$. From it we obtain $d-2 g-1$ further equations $\phi_{m}$, by rolling factors. Write $U(x)=\sum_{i=0}^{g+1-e} U_{i} x^{i}, V(x)=\sum_{i=0}^{g-e} V_{i} x^{i}$ and $W(x)=\sum_{i=0}^{g+1+e} W_{i} x^{i}$; this defines $F$. Then we have the following equations:

$$
\begin{aligned}
& \operatorname{Rank}\left(\begin{array}{ccccccc}
z_{0} & z_{1} & \ldots & z_{k-1} & w_{0} & \ldots & w_{k-e-1} \\
z_{1} & z_{2} & \ldots & z_{k} & w_{1} & \ldots & w_{k-e}
\end{array}\right) \leq 1 \\
& \phi_{m}=\sum_{i=0}^{g+1-e} U_{i} w_{\left\lfloor\frac{m+i}{2}\right\rfloor} w_{\left\lceil\frac{m+i}{2}\right\rceil}-2 \sum_{i=0}^{g-e} V_{i} w_{\left\lfloor\frac{m+i}{2}\right\rfloor} z_{\left\lceil\frac{m+i}{2}\right\rceil}-\sum_{i=0}^{g+1+e} W_{i} z_{\left\lfloor\frac{m+i}{2}\right\rfloor} z_{\left\lceil\frac{m+i}{2}\right\rceil} \text {, } \\
& m=0, \ldots, d-2 g-2 \text {. }
\end{aligned}
$$

(2.3) Rolling factors format [Reid 1989]. This format occurs often in connection with divisors on scrolls. We start with a $k$-dimensional rational normal scroll $S \in \mathbb{P}=\mathbb{P}^{n}$; the classical construction is to take $k$ complementary linear subspaces $L_{i}$, spanning $\mathbb{P}$, with a parametrised rational normal curve $\phi_{i}: \mathbb{P}^{1} \rightarrow C_{i} \subset L_{i}$ of degree $d_{i}=\operatorname{dim} L_{i}$ in it, and to take for each $p \in \mathbb{P}^{1}$ the span of the points $\phi_{i}(p)$. 
The degree of $S$ is $f=\sum d_{i}$. The scroll $S$ can be defined by the minors of the matrix:

$$
\left(\begin{array}{ccccccc}
z_{0}^{(1)} & \ldots & z_{d_{1}-1}^{(1)} & \ldots & z_{0}^{(k)} & \ldots & z_{d_{k}-1}^{(k)} \\
z_{1}^{(1)} & \ldots & z_{d_{1}}^{(1)} & \ldots & z_{1}^{(k)} & \ldots & z_{d_{k}}^{(k)}
\end{array}\right) .
$$

The Picard group of $S$ is generated by the hyperplane class $H$ and the ruling $R$ [Schreyer 1986, Sect. 1]; let $C$ be a divisor of type $a H-b R$. The resolution of $\mathcal{O}_{C}$ as $\mathcal{O}_{S}$-module is $0 \rightarrow \mathcal{O}_{S}(-a H+b R) \rightarrow \mathcal{O}_{S} \rightarrow \mathcal{O}_{C} \rightarrow 0$. Schreyer describes, following Eisenbud, Eagon-Northcott type complexes $\mathcal{C}^{b}$ such that $\mathcal{C}^{b}(a)$ is the minimal resolution of $\mathcal{O}_{S}(-a H+b R)$ as $\mathcal{O}_{\mathbb{P}}$-module, if $b \geq-1$ [loc. cit., 1.2]. The resolution of $\mathcal{O}_{C}$ is then obtained by taking a mapping cone. We obtain the following first terms of the resolution:

$$
\begin{aligned}
\left(\mathcal{O}_{\mathbb{P}}^{f}(-1) \otimes \operatorname{Sym}_{b-1} \mathcal{O}_{\mathbb{P}}^{2}\right) & (-a) \oplus \wedge^{3} \mathcal{O}_{\mathbb{P}}^{f}(-1) \otimes \mathcal{O}_{\mathbb{P}}^{2} \longrightarrow \\
\longrightarrow & \left.\longrightarrow \operatorname{Sym}_{b} \mathcal{O}_{\mathbb{P}}^{2}\right)(-a) \oplus \wedge^{2} \mathcal{O}_{\mathbb{P}}^{f}(-1) \longrightarrow \mathcal{O}_{\mathbb{P}} \longrightarrow \mathcal{O}_{C} \rightarrow 0 .
\end{aligned}
$$

The $b+1$ equations $\phi_{m}$, describing $C$ on $S$, are obtained by 'rolling' along $\mathbb{P}^{1}$ : in terms of an inhomogeneous coordinate $x$ on $\mathbb{P}^{1}$ we have $z_{j}^{(i)}=\psi^{(i)} x^{j}$ for some function $\psi^{(i)}$, and in the transition from the equation $\phi_{m}$ to $\phi_{m+1}$, which is obtained by multiplying with $x$, we have to increase the lower index by one for exactly one of the factors $z_{j}^{(i)}$ in each monomial.

(2.4) Infinitesimal deformations. For the cone $X$ over a hyperelliptic curve of degree at least $2 g+3$, the dimension of $T_{X}^{1}(-1)$ is $2 g+2$, by Proposition (1.25). We want to describe the action on the equations. For this we have to study the normal bundle of $C$.

Let $d=\operatorname{deg} L \geq 2 g+3$. For the scroll $S=\mathbb{P}\left(\pi_{*} L\right)$, we have $\mathcal{O}_{S}(1) \sim E_{0}+a f$, so the divisor of the line bundle $N_{C / S}(-1)$ is $C \cdot(L+(2 g+2-d) f)$. From the exact sequence:

$$
0 \rightarrow H^{0}\left(N_{C / S}(-1)\right) \longrightarrow H^{0}\left(N_{C}(-1)\right) \longrightarrow H^{0}\left(\left.N_{S}\right|_{C}(-1)\right) \longrightarrow H^{1}\left(N_{C / S}(-1)\right)
$$

we obtain that $h^{0}\left(C,\left.N_{S}\right|_{C}(-1)\right) \leq h^{0}\left(C, N_{C}(-1)\right)-\chi\left(N_{C / S}(-1)\right)=d+g+3-$ $(3 g+5-d)=2 d-2 g-2$, where $h^{0}\left(C, N_{C}(-1)\right)$ can be computed with the sequence $0 \longrightarrow V^{*} \otimes H^{0}\left(C, \mathcal{O}_{C}\right) \longrightarrow H^{0}\left(C, N_{C}(-1)\right) \longrightarrow T_{X}^{1}(-1) \longrightarrow 0$, by Theorem (1.4). On the other hand, $h^{0}\left(S, N_{S}(-1)\right)=2 d-2 g-2$, and all deformations are obtained by deforming the matrix of the scroll; because $H^{0}\left(C, \mathcal{O}_{C}(1)\right)=H^{0}\left(S, \mathcal{O}_{S}(1)\right)$, the restriction map $H^{0}\left(S, N_{S}(-1)\right) \rightarrow H^{0}\left(C,\left.N_{S}\right|_{C}(-1)\right)$ is injective and so for dimension reasons an isomorphism. This shows that every infinitesimal deformation of negative weight of $X$ comes from a deformation of the scroll.

We only compute the case $L=k g_{2}^{1}$; then $N_{C / S}(-1)=(2 g+2-k) g_{2}^{1}$.

(2.5) Proposition. Let $L=k g_{2}^{1}$ with $k>g+1$. On $T^{1}(-1)$ we take coordinates $\left(s_{1}, \ldots, s_{k-1}, t_{0}, \ldots, t_{2 g+2-k}\right)$, subject to the relations $\sum_{i=0}^{2 g+2} a_{i} s_{i+j}=0$ for $j=1$, $\ldots, k-2 g-3$. The first order deformations are the given by:

$$
\begin{gathered}
\operatorname{Rank}\left(\begin{array}{ccccccc}
z_{0} & z_{1}+s_{1} & \ldots & z_{k-1}+s_{k-1} & w_{0} & \ldots & w_{k-g-2} \\
z_{1} & z_{2} & \ldots & z_{k} & w_{1} & \ldots & w_{k-g-1}
\end{array}\right) \leq 1, \\
\phi_{m}+\sum_{i=0}^{2 g+2-k} t_{i} z_{m+i}-\sum_{j=0}^{2 g+2} \sum_{i=m+j}^{2 g+2} a_{i} s_{i-\left\lceil\frac{j}{2}\right\rceil} z_{m+\left\lceil\frac{j}{2}\right\rceil}+\sum_{j=1}^{m-1} \sum_{i=0}^{m-j-1} a_{i} s_{i+\left\lfloor\frac{j}{2}\right\rfloor} z_{m-\left\lfloor\frac{j}{2}\right\rfloor},
\end{gathered}
$$


where $\phi_{m}$ is as above, $m=0, \ldots, 2 k-2 g-2$. Furthermore, $a_{j}=0$ for $j>2 g+2$, $s_{0}=0$ and $s_{j}=0$ for $j \geq k$. Therefore in the last term the summation really runs to $\min (2 g+2, m-j-1)$, and in the other term to $\min \left(2 g+2, k-1+\left\lceil\frac{j}{2}\right\rceil\right)$.

Proof. Consider the following relation, involving $\phi_{m}$ :

$$
\begin{aligned}
z_{j+1} \phi_{m}-z_{j} \phi_{m+1}+w_{\left\lceil\frac{m}{2}\right\rceil}\left(z_{j} w_{\left\lceil\frac{m+1}{2}\right\rceil}-z_{j+1} w_{\left\lfloor\frac{m}{2}\right\rfloor}\right) & \\
& -\sum_{i=0}^{2 g+2} a_{i} z_{\left\lceil\frac{m+i}{2}\right\rceil}\left(z_{j} z_{\left\lceil\frac{m+i+1}{2}\right\rceil}-z_{j+1} z_{\left\lfloor\frac{m+i}{2}\right\rfloor}\right)=0 .
\end{aligned}
$$

Now deform $\phi_{m}$ to $\phi_{m}+\phi_{m}^{\prime}$, and the other equations as given by the matrix. The condition that this defines an infinitesimal deformation, is that in the local ring of the singularity the following equation holds:

$$
z_{j+1} \phi_{m}^{\prime}-z_{j} \phi_{m+1}^{\prime}+\sum_{i=0}^{2 g+2} a_{i} s_{\left\lfloor\frac{m+i}{2}\right\rfloor} z_{j} z_{\left\lceil\frac{m+i}{2}\right\rceil}=0 .
$$

In terms of the inhomogeneous coordinates $(x, y)$ we can write:

$$
x \phi_{m}^{\prime}-\phi_{m+1}^{\prime}+x \sum_{i=0}^{2 g+2} a_{i} s_{\left\lfloor\frac{m+i}{2}\right\rfloor} x^{\left\lceil\frac{m+i}{2}\right\rceil}=0 .
$$

This equation is independent of $j$. The relations involving $w_{j+1} \phi_{m}$ give the same set of equations. It is easily checked that the given formula for $\phi_{m}^{\prime}$ satisfies this set of equations. Furthermore, it gives a well defined element of the local ring: we check that no $z_{i}$ with $i>k$ occurs with non-zero coefficient. If in the last term $m-j-1<2 g+2$, then $\left\lfloor\frac{j}{2}\right\rfloor \geq\left\lfloor\frac{m}{2}\right\rfloor-g-1$ and $m-\left\lfloor\frac{j}{2}\right\rfloor \leq\left\lceil\frac{m}{2}\right\rceil+g+1 \leq k$, because $m \leq 2 k-2 g-2$. Likewise, if $m+j \leq 2 g+2$, then $m+\left\lceil\frac{j}{2}\right\rceil \leq k$.

(2.6) $T^{2}$. To determine the dimension of $T^{2}$ we use the MAIN LEMMA of [BehnkeChristophersen 1991, 1.3.2]. Let $h: X \rightarrow \mathbb{C}$ define a hyperplane section $Y=h^{-1}(0)$ of $X$, then $\operatorname{dim} T_{X}^{2} / h T_{X}^{2}=\operatorname{dim} T_{Y}^{1}-e_{h}$, where $e_{h}$ is the dimension of the smoothing component, on which the smoothing $h$ of $Y$ lies. A general hyperplane section $Y$ is the cone over $d$ points in $\mathbb{P}^{d-g-1}$, lying on a rational normal curve of degree $d-g-1$. Equations for this curve singularity can of course also be written in rolling factors format: let the polynomial $F(\bar{x}, x)=\sum_{i=0}^{d} a_{i} \bar{x}^{d-i} x^{i}$ determine the points on the rational curve, then we have:

$$
\begin{gathered}
\operatorname{Rank}\left(\begin{array}{cccc}
z_{0} & z_{1} & \ldots & z_{d-g-2} \\
z_{1} & z_{2} & \ldots & z_{d-g-1}
\end{array}\right) \leq 1, \\
\phi_{m}=\sum_{i=0}^{d} a_{i} z_{\left\lfloor\frac{m+i}{2}\right\rfloor} z_{\left\lceil\frac{m+i}{2}\right\rceil}, \quad m=0, \ldots, d-2 g-2 .
\end{gathered}
$$

(2.7) Lemma. The $\mathcal{O}_{Y}$-module $T_{Y}^{2}$ is annihilated by the maximal ideal $\mathfrak{m}_{Y}$. 
Proof. Let $N=\left(\begin{array}{c}d-g-1 \\ 2\end{array}\right)+d-2 g-1$ be the number of equations. We want to show that $h \psi \in \operatorname{Im} \operatorname{Hom}\left(\left(\mathcal{O}_{X}\right)^{\oplus k}, \mathcal{O}_{X}\right)$ for every $\psi \in \operatorname{Hom}\left(R / R_{0}, \mathcal{O}_{X}\right)$ and every $h \in \mathfrak{m}_{X}$. In the determinantal case this is shown in [Behnke-Christophersen 1991, 2.1.1]; we remark that the proof as written is not correct: some equalities do not hold modulo $R_{0}$, but only modulo the larger submodule $R_{I}$ of relations with entries in the ideal $I$ of $X$; this is not a serious problem, because $\operatorname{Hom}\left(R / R_{I}, \mathcal{O}_{X}\right)=$ $\operatorname{Hom}\left(R / R_{0}, \mathcal{O}_{X}\right)$. Using their computations we may assume that $h \psi$ vanishes on determinantal relations.

To describe the additional relations, we introduce the notation $f_{i, j}=z_{i} z_{j+1}-$ $z_{i+1} z_{j}$. We get:

$$
R_{j, m}=\phi_{m+1} z_{j}-\phi_{m} z_{j+1}-\sum_{i=0}^{d} a_{i} f_{j,\left\lfloor\frac{m+i}{2}\right\rfloor} z_{\left\lceil\frac{m+i}{2}\right\rceil}
$$

where $0 \leq j<d-g-1$ and $0 \leq m<d-2 g-2$. The determinantal relations are $R_{i, j, k}=f_{i, j} z_{k}-f_{i, k} z_{j}+f_{j, k} z_{i}$ and $S_{i, j, k}=f_{i, j} z_{k+1}-f_{i, k} z_{j+1}+f_{j, k} z_{i+1}$. We have the following equality:

$$
R_{j, m} z_{k}-R_{k, m} z_{j}-\sum_{i=0}^{d} a_{i} R_{j, k,\left\lfloor\frac{m+i}{2}\right\rfloor} z_{\left\lceil\frac{m+i}{2}\right\rceil}=\phi_{m} j\left(f_{j, k}\right)-f_{j, k} j\left(\phi_{m}\right) .
$$

Now fix an element $z_{j} \in \mathfrak{m}_{Y}$. We look for $\psi_{m} \in \mathcal{O}_{Y}$ with $\psi\left(z_{j} R_{k, m}\right)=\psi_{m+1} z_{k}-$ $\psi_{m} z_{k+1}$ for all $k$ and $m$. Because $\psi\left(z_{j} R_{k, m}\right)=z_{k} \psi\left(R_{j, m}\right)$, we can determine the $\psi_{m}$ from the $\psi\left(R_{j, m}\right)$, with the equations $\bar{x} \psi\left(R_{j, m}\right)-\bar{x} \psi_{m+1}+x \psi_{m}$; take $\psi_{j}=0$ and solve. For $m>j$ we get that $\bar{x}^{m-j} \psi_{j, m}=\bar{x}^{m-j-1} x \psi\left(R_{j, m-1}\right)+$ $\cdots+x^{m-j} \psi\left(R_{j, j}\right)$; because $z_{j+k} \psi\left(R_{j, l}\right)=z_{j} \psi\left(R_{j+k, l}\right)$ we can divide by $\bar{x}^{m-j}$. A similar argument shows that we can solve for $\psi_{m}$ with $m<j$.

Important for our application is that the corresponding result holds for $T_{X}^{2}$; the computation is similar to the one for the hyperplane section, but the formula's are more complicated. I expect that there is a general statement for the rolling factors format, but it is not quite clear what the 'generic' rolling factors singularity is.

We introduce more notation: $g_{i, j}=z_{i} w_{j+1}-z_{i+1} w_{j}$ and $h_{i, j}=w_{i} w_{j+1}-$ $w_{i+1} w_{j}$. We have a relation $R_{j, m}$ :

$$
\begin{aligned}
R_{j, m}=\phi_{m+1} z_{j}-\phi_{m} z_{j+1} & -\sum U_{i} g_{j,\left\lfloor\frac{m+i}{2}\right\rfloor} w_{\left\lceil\frac{m+i}{2}\right\rceil}+2 \sum V_{i} f_{j, \frac{m+i}{2}} w_{\frac{m+i}{2}} \\
& +2 \sum V_{i} g_{j, \frac{m+i-1}{2}} z_{\frac{m+i+1}{2}}+\sum W_{i} f_{j,\left\lfloor\frac{m+i}{2}\right\rfloor}{ }_{\left\lceil\frac{m+i}{2}\right\rceil},
\end{aligned}
$$

where one term with $V_{i}$ has to be chosen, depending on the parity of $m+i$; we have similar relations $S_{j, m}$, involving $w_{j}$ and $w_{j+1}$. We find as above that $R_{j, m} z_{k}-R_{k, m} z_{j} \equiv 0 \quad\left(\bmod R_{D}\right)$, where $R_{D}$ is the submodule, generated by the trivial and determinantal relations. In the same way $S_{j, m} z_{k}-R_{k, m} w_{j} \equiv 0$ $\left(\bmod R_{D}\right)$ and $S_{j, m} w_{k}-S_{k, m} w_{j} \equiv 0 \quad\left(\bmod R_{D}\right)$. This gives the desired result:

(2.8) Lemma. The module $T_{X}^{2}$ is annihilated by the maximal ideal. 
(2.9) Proposition. Let $Y$ be the cone over $d$ points on the rational normal curve of degree $d-g-1$, with $d<2(d-g-1)$. Then $\operatorname{dim} T_{Y}^{1}(-1)=d$, $\operatorname{dim} T_{Y}^{1}(0)=$ $(g-1)(d-g-1)$, and $\operatorname{dim} T_{Y}^{1}(\nu)=0$ for $\nu \neq 0,-1$.

Proof. The $d$ points are in generic position [Greuel 1982, 3.3]: a set of $d$ points in $\mathbb{P}^{k}$ is in general position, if for every $n$ the images of the points under the $n$-tuple Veronese embedding of $\mathbb{P}^{k}$ span a linear space of maximal possible dimension, i.e. of dimension $\min \left\{d, \frac{k+n}{n}\right\}-1$. For points on a rational normal curve of degree $k$ in $\mathbb{P}^{k}$ we have to consider the composed $k n$-tuple embedding of $\mathbb{P}^{1}$; the dimension of the span is $\min \{d, k n\}-1$. In our case $d<2 k$, so every subset of the $d$ points is in general position, and by definition the $d$ points are in generic position. Therefore $Y$ is negatively graded in the sense of [Pinkham 1970], cf. [Greuel 1982, 3.3], so $\operatorname{dim} T_{Y}^{1}(\nu)=0$ for $\nu>0$.

The dimension of $T_{Y}^{1}(0)$ is equal to the number of moduli for $d$ points in $\mathbb{P}^{d-g-1}$. Because every (quadratic) equations occurs in a linear relation, $T_{Y}^{1}$ vanishes in degree $<-1$. We finish the proof by computing $T_{Y}^{1}(-1)$ in three steps.

Step 1. Every infinitesimal deformation of $Y$ of degree -1 comes from a deformation of the cone over the rational normal curve of degree $d-g-1$.

Proof. We may assume that $(\bar{x}, x)=(1,0)$ or $(0,1)$ is not a root of the polynomial $F$, i.e. $a_{0} \neq 0$ and $a_{d} \neq 0$. Then $\bar{x}$ and $x$ are not zero divisors in $\mathcal{O}_{Y}$. Therefore we get from the determinantal relations the equations:

$$
f_{j, j+k} \bar{x}^{k-1} x=f_{j+1, j+k} \bar{x}^{k}+f_{j, j+1} x^{k} .
$$

By induction we find:

$$
f_{j, j+k} \bar{x}^{k-1} x^{k-1}=\sum_{i=0}^{k-1} f_{j+i, j+i+1} \bar{x}^{2 i} x^{2(k-i-1)} .
$$

Let $n \in \operatorname{Hom}\left(I / I^{2}, \mathcal{O}_{Y}\right)$ be a normal vector of degree -1 with $n\left(f_{i, i+1}\right)=g_{i}$. From equation $\left(*_{j, k}\right)$ we get that $n\left(f_{j, j+k}\right) \bar{x}^{k-1} x^{k-1}=\sum g_{j+i} \bar{x}^{2 i} x^{2(k-i-1)}$. We claim that $g_{j+i}$ is divisible by $\bar{x}^{k-i-1} x^{i}$. Every $g_{j+i}$ occurs in $\left(*_{j+1, k}\right)$ or $\left(*_{j, k-1}\right)$, so by induction we obtain $g_{j}=\bar{x}^{k-2} g_{j}^{\prime}, g_{j+k-1}=x^{k-2} g_{j+k-1}^{\prime}$ and the claim for $0<i<k-2$. Therefore we have $x^{2} g_{j}^{\prime}+\bar{x}^{2} g_{j+k-1}^{\prime}-\bar{x} x \psi \equiv 0 \quad(\bmod F)$ for some polynomial $\psi(\bar{x}, x)$. The degree of $x^{2} g_{j}^{\prime}(\bar{x}, x)$ is at most $d-g+1$; because $g>1$, we have in fact a polynomial equation in $\bar{x}$ and $x$, so $g_{j}^{\prime}$ is divisible by $\bar{x}$, and $g_{j+k-1}^{\prime}$ by $x$. By taking $k$ maximal we see that $g_{j}=\lambda_{j} z_{j}+\mu_{j} z_{j+1}+\nu_{j} z_{j+2}$ for some $\lambda_{j}, \mu_{j}, \nu_{j} \in \mathbb{C}$ : this is the formula for the infinitesimal deformations of the cone over the rational normal curve.

Step 2. The following formula's define a $d$-dimensional subspace of $T_{Y}^{1}(-1)$; deformation parameters are $\left(s_{1}, \ldots, s_{d-g-2}, t_{0}, \ldots, t_{g+1}\right)$.

$$
\operatorname{Rank}\left(\begin{array}{cccc}
z_{0} & z_{1}+s_{1} & \ldots & z_{d-g-2}+s_{d-g-2} \\
z_{1} & z_{2} & \ldots & z_{d-g-1}
\end{array}\right) \leq 1
$$




$$
\begin{aligned}
\phi_{m}+\sum_{i=0}^{g+1} t_{i} z_{m+i}+\sum_{i=1}^{d-g-2} \sum_{j=0}^{2 i-2 m-1} s_{i} a_{i+1+\left\lceil\frac{j}{2}\right\rceil^{z}} z_{m+1+\left\lceil\frac{j}{2}\right\rceil} & -\sum_{i=1}^{d-g-2} \sum_{j=0}^{2 m-2 i-1} s_{i} a_{2 i+1-m+\left\lceil\frac{j}{2}\right\rceil} z_{i+1+\left\lceil\frac{j}{2}\right\rceil},
\end{aligned}
$$

where $\phi_{m}$ is as above, $m=0, \ldots, 2 k-2 g-2$. Furthermore, $a_{j}=0$ for $j>d$ or $j<0$.

Proof. The deformation of the matrix gives the equation $f_{j, k}+s_{j} z_{k+1}-s_{k} z_{j+1}$. Suppose $\phi_{m}$ is deformed to $\phi_{m}+\phi_{m}^{\prime}$. The relations $R_{j, m}$ give:

$$
\bar{x} \phi_{m+1}^{\prime}-x \phi_{m}^{\prime}+\sum_{i=0}^{d} a_{i} s_{\left\lfloor\frac{m+i}{2}\right\rfloor} x^{\left\lceil\frac{m+i}{2}\right\rceil+1}=0 .
$$

The given formula satisfies these equations. The indices in it do not exceed $d-g-1$.

Step 3. The deformations of the scroll, which deform $f_{j, j+1}$ by $g_{j}=\mu_{j} z_{j+1}$, do not extend to deformations of $Y$.

Proof. Suppose $f_{i, j}$ and $\phi_{m}$ deform with $g_{i, j}$ and $\phi_{m}^{\prime}$. From equation $\left(*_{j, k}\right)$ we get that $g_{j, j+k}=\sum_{i=j}^{j+k-1} \mu_{i} x^{2 j+k-i}$. The relation $R_{j, m}$ gives that

$$
\phi_{m+1}^{\prime}-x \phi_{m}^{\prime}+\sum_{i=0}^{2 j-m-1} a_{i} \sum_{k=\left\lfloor\frac{m+i}{2}\right\rfloor}^{j-1} \mu_{k} x^{m+i-k}-\sum_{i=2 j+2-m}^{d} a_{i} \sum_{k=j}^{\left\lfloor\frac{m+i}{2}\right\rfloor-1} \mu_{k} x^{m+i-k}=0 .
$$

Because $F(\bar{x}, x)=0$, this expression is independent of $j$, so we take $j=0$. Let $\alpha$ be the smallest index such that $\mu_{\alpha} \neq 0$. We set $m=2 d-2 g-3$ :

$$
\phi_{2 d-2 g-2}^{\prime}-x \phi_{2 d-2 g-3}^{\prime}-\sum_{i=0}^{d} \sum_{k=g+3-\left\lceil\frac{d}{2}\right\rceil}^{i-\alpha} a_{i} \mu_{i-k} x^{2 d-2 g-3+k}=0 .
$$

Because $d-g-1$ is the highest power of $x$, which can occur in $\phi_{2 d-2 g-3}^{\prime}$, the coefficient of $3 d-2 g-3-\alpha \geq 2 d-g-1$ in the sum vanishes; so $a_{d} \mu_{\alpha}=0$, which contradicts our assumption that $a_{d} \neq 0$. Therefore all $\mu_{i}$ are zero.

(2.10) Proposition. Let $X=X(C, L)$ be the cone over a hyperelliptic curve of genus $g$, embedded with a complete linear system $L$ of degree $d$, with $d>2 g+3$. Then $\operatorname{dim} T_{X}^{2}(-2)=d-2 g-3, \operatorname{dim} T_{X}^{2}(-1)=(g-2)(d-g-3)$, and $\operatorname{dim} T_{X}^{2}(\nu)=0$ for $\nu \neq-1,-2$.

Proof. For the hyperplane section $Y$ the previous Proposition gives $\operatorname{dim} T_{Y}^{1}=$ $d+(g-1)(d-g-1)$; the dimension of a smoothing component is $e=\mu+t-1$ [Greuel 1982, 2.5.(3)], where $t$ is the type of the singularity. In our case $t=g=$ $\delta(Y)-d+1$, so $e=3 g+d-2$. Because $T_{X}^{2}$ is annihilated by the maximal ideal, the Main Lemma of [Behnke-Christophersen 1991] gives $\operatorname{dim} T_{X}^{2}=\operatorname{dim} T_{Y}^{1}-e=$ $(g-1)(d-g-4)-1$. By the formula of $(1.26)$ we have $T_{X}^{2}(-1)=(g-2) h^{0}(L)-$ $6(g-1)+\operatorname{dim} T_{X}^{1}(-1)$. By Lemma $(1.28), \operatorname{dim} T_{X}^{2}(\nu)=0$ for $\nu<-1$. 
(2.11) Remark. One can also compute $T_{X}^{2}(-2)$ directly; we sketch this here for the special case $L=k g_{2}^{1}$. We have to show that $\operatorname{dim} \operatorname{ker}\left\{H^{0}(L) \otimes H^{1}\left(L^{-1}\right) \rightarrow\right.$ $\left.H^{1}\left(\mathcal{O}_{C}\right)^{\oplus N}\right\}=10 k+2 g-7$, where $N$ is the number of equations. The elements $y /\left(\bar{x}^{g+1-i} x^{i}\right), i=1, \ldots, g$, form a basis of $H^{1}\left(\mathcal{O}_{C}\right)$; we abbreviate $[i]=y /\left(\bar{x}^{g+1-i} x^{i}\right)$, for all $i \in \mathbb{Z}$. A basis of $H^{0}(L) \otimes H^{1}\left(L^{-1}\right)$ is:

$$
\begin{array}{rlrl}
\phi_{i, j} & =\frac{1}{\bar{x}^{k-i} x^{i}} \frac{\partial}{\partial z_{j}} & i=1, \ldots, k-1, \quad j=0, \ldots, k, \\
\psi_{i, j}=\frac{y}{\bar{x}^{g+k+1-i} x^{i}} \frac{\partial}{\partial z_{j}} & i=1, \ldots, g+k, \quad j=0, \ldots, k, \\
\chi_{i, j}=\frac{1}{\bar{x}^{k-i} x^{i}} \frac{\partial}{\partial w_{j}} & i=1, \ldots, k-1, \quad j=0, \ldots, k-g-1, \\
\theta_{i, j}=\frac{y}{\bar{x}^{g+k+1-i} x^{i}} \frac{\partial}{\partial w_{j}} & i=1, \ldots, g+k, \quad j=0, \ldots, k-g-1 .
\end{array}
$$

On $f_{l, m}$ only the action of $\psi_{i, j}$ is non trivial: $\psi_{i, l}\left(f_{l, m}\right)=[i-m-1], \psi_{i, l+1}\left(f_{l, m}\right)=$ $-[i-m], \psi_{i, m+1}\left(f_{l, m}\right)=[i-l], \psi_{i, m}\left(f_{l, m}\right)=[i-l-1]$ and $\psi_{i, j}\left(f_{l, m}\right)=0$ otherwise. Therefore:

$$
\sum_{i, j} \alpha_{i, j} \psi_{i, j}\left(f_{l, m}\right)=\sum_{i}\left(\alpha_{i+m+1, l}+\alpha_{i+l, m+1}-\alpha_{i+m, l+1}-\alpha_{i+l+1, m}\right)[i] .
$$

The coefficient of $[i]$ has to vanish for $1 \leq i \leq g$. A set of solutions to these equations is given by $\alpha_{i, j}=\alpha_{i+j}$ for some constants $\alpha_{j}, j=1, \ldots, g+2 k$. Define $\beta_{i, j}=\alpha_{i+1, j}-\alpha_{i, j+1}$, where $i=1, \ldots, g+k-1$ and $j=0, \ldots, k-1$. We have the equations $\beta_{i+m, l}=\beta_{i+l, m}$ for $i=1, \ldots, g$ and $0 \leq l<m \leq k-1$. Consider pairs $(i, j)$ and $\left(i^{\prime}, j^{\prime}\right)$ with $i+j=i^{\prime}+j^{\prime}$, and suppose that $j=j^{\prime}+s(g-1)+(g-r)$. Write $(i, j) \sim\left(i^{\prime}, j^{\prime}\right)$ if $\beta_{i, j}=\beta_{i^{\prime}, j^{\prime}}$. If $i-j^{\prime}>g$, we can use the equations to obtain $(i, j) \sim(t+j, i-t) \sim(i+g-t, j-g+t)$, where $t=r$ if $s=0$, or $t=1$ for $s>0$; for $i-j^{\prime}<0$ we have a similar inductive procedure, whereas otherwise a suitable equation directly gives that $(i, j) \sim\left(i^{\prime}, j^{\prime}\right)$ if $i+j=i^{\prime}+j^{\prime}$.

For the action of $\chi_{i, j}$ on $h_{l, m}$ we have analogous computations. The next step is to compute the action on $\phi_{m}$ :

$$
\begin{aligned}
& \sum\left(\left(\alpha_{i+j}+j \beta_{i+j}\right) \psi_{i, j}+\left(\gamma_{i+j}+j \delta_{i+j}\right) \chi_{i, j}\right)\left(\phi_{m}\right)= \\
& =\sum\left(\gamma_{i+\left\lfloor\frac{m}{2}\right\rfloor}+\left\lfloor\frac{m}{2}\right\rfloor \delta_{i+\left\lfloor\frac{m}{2}\right\rfloor}\right)\left[\left\lceil\frac{m}{2}\right\rceil-i\right]+\left(\gamma_{i+\left\lceil\frac{m}{2}\right\rceil}+\left\lceil\frac{m}{2}\right\rceil \delta_{i+\left\lceil\frac{m}{2}\right\rceil}\right)\left[\left\lfloor\frac{m}{2}\right\rfloor-i\right] \\
& \quad-a_{l}\left(\alpha_{i+\left\lfloor\frac{m+l}{2}\right\rfloor}+\left\lfloor\frac{m+l}{2}\right\rfloor \beta_{i+\left\lfloor\frac{m+l}{2}\right\rfloor}\right)\left[\left\lceil\frac{m+l}{2}\right\rceil-i\right] \\
& \quad-a_{l}\left(\alpha_{i+\left\lceil\frac{m+l}{2}\right\rceil}+\left\lceil\frac{m+l}{2}\right\rceil \beta_{i+\left\lceil\frac{m+l}{2}\right\rceil}\right)\left[\left\lfloor\frac{m+l}{2}\right\rfloor-i\right] \\
& =\sum\left(2 \gamma_{m-c}+m \delta_{m-c}-2 a_{l} \alpha_{m+l-c}-a_{l}(m+l) \beta_{m+l-c}\right)[c]
\end{aligned}
$$

We get equations $\delta_{j}-\sum_{i=0}^{2 g+2} a_{i} \beta_{j+i}$ and $2 \gamma_{j}-\sum_{i=0}^{2 g+2} a_{i}\left(2 \alpha_{j+i}-i \beta_{j+i}\right)$, except for $j=2 k-g-2$, when there is only one equation; but there is no $\delta_{2 k-g-2}$. So the parameters $\gamma_{j}$ and $\delta_{j}$ are completely determined by $\alpha_{j}$ and $\beta_{j}$. As $\beta_{1}=\beta_{2 k+g}=0$, we have a $(4 k+2 g-2)$-dimensional solution space. 
Finally, we compute the action on $z_{l} w_{m+1}-z_{l+1} w_{m}$ :

$\sum\left(\alpha_{i, j} \phi_{i, j}+\beta_{i, j} \theta_{i, j}\right)\left(g_{l, m}\right)=\sum_{i}\left(\alpha_{i+m+1, l}+\beta_{i+l, m+1}-\alpha_{i+m, l+1}-\beta_{i+l+1, m}\right)[i]$.

The coefficient of $[i]$ certainly vanishes, if $\alpha_{i, j}$ and $\beta_{i, j}$ depend only on $i+j$. Define $\gamma_{i, j}=\alpha_{i+1, j}-\alpha_{i, j+1}$ and $\delta_{i, j}=\beta_{i+1, j}-\beta_{i, j+1}$. Then $\gamma_{i+m, l}=\delta_{i+l, m}$. One shows that $\gamma_{i, j}=\delta_{i, j}=\epsilon_{i+j}$ for all $i, j$. The solution space has dimension $6 k-5$.

The dimension of $T_{X}^{2}(-2)$ is two less than the number of equations $\phi_{m}$. This fact has an interpretation in terms of the rolling factors format, if all the $\phi_{m}$ are quadratic equations.

(2.12) Proposition. Let $X$ be the cone over a divisor of type $2 H-b R$ on a scroll $S$. Consider infinitesimal deformations of negative degree of $X$, which come from deformations of the cone over $S$; if the versal deformation of the cone over $S$ is:

$$
\left(\begin{array}{cccccccc}
z_{0}^{(1)} & z_{1}^{(1)}+s_{1}^{(1)} & \ldots & z_{d_{1}-1}^{(1)}+s_{d_{1}-1}^{(1)} & \ldots & z_{0}^{(k)} & \ldots & z_{d_{k}-1}^{(k)}+s_{d_{k}-1}^{(k)} \\
z_{1}^{(1)} & z_{2}^{(1)} & \ldots & z_{d_{1}}^{(1)} & \ldots & z_{1}^{(k)} & \ldots & z_{d_{k}}^{(k)}
\end{array}\right)
$$

then there are additional parameters $t_{i}$, and linear equations on the $t_{i}$ and $s_{i}^{(j)}$, such that the additional equations are given by $\phi_{m}+\phi_{m}^{\prime}(\boldsymbol{t}, \boldsymbol{s}, \boldsymbol{z}), m=0, \ldots, b$. Then this deformation can be extended over a base space given by $b-1$ equations $\phi_{m}^{\prime}(\boldsymbol{t}, \boldsymbol{s}, \boldsymbol{s})-\phi_{m}(\boldsymbol{s})=0, m=1, \ldots, b-1$.

Proof. We have to lift the relations involving the $\phi_{m}$. The rolling factors assumption gives that we can write $\phi_{m}=\sum_{\alpha} z_{\alpha} c_{\alpha}$ and $\phi_{m+1}=\sum_{\alpha} z_{\alpha+1} c_{\alpha}$, where $c_{\alpha}$ depends (linearly) on $z$, and $\alpha$ runs through all possible indices ${ }_{j}^{(i)}$, and $c_{\alpha}$ depends (linearly) on $\boldsymbol{z}$. This gives the relation $\phi_{m+1} z_{j}^{(i)}-\phi_{m} z_{j+1}^{(i)}-\sum f_{j \alpha}^{(i)} c_{\alpha}$, where $f_{j \alpha}^{(i)}$ is the determinantal equation $z_{j}^{(i)} z_{\alpha+1}-z_{j+1}^{(i)} z_{\alpha}$. We lift it to:

$$
\begin{aligned}
\left(\phi_{m+1}+\phi_{m+1}^{\prime}(\boldsymbol{z})\right)\left(z_{j}^{(i)}+s_{j}^{(i)}\right)-\left(\phi_{m}+\phi_{m}^{\prime}(\boldsymbol{z}+\boldsymbol{s})\right) z_{j+1}^{(i)}-\sum \tilde{f}_{j, \alpha}^{(i)} c_{\alpha} & \\
& \equiv \sum z_{j+1}^{(i)} s_{\alpha} c_{\alpha}(\boldsymbol{s}) \quad(\bmod \mathcal{I})
\end{aligned}
$$

where $\mathcal{I}$ is the ideal of the deformed scroll, and $\tilde{f}_{j \alpha}^{(i)}$ is a deformed equation. The lift up to first order is possible by assumption. If $1 \leq m \leq b-1$, then $\phi_{m}$ occurs in a relation as first and as second term. Therefore $\phi_{m}^{\prime}(\boldsymbol{z})$ and $\phi_{m}^{\prime}(\boldsymbol{z}+\boldsymbol{s})-\phi_{m}(\boldsymbol{z})$ have to be equal.

(2.13) Example. Let $X$ be the cone over a hyperelliptic curve, embedded with $(2 g+2) g_{2}^{1}$. With Proposition (2.5) we find as equations for the negative degree part of the versal base:

$$
t s_{m}-\sum_{\substack{i=m+j \\ j=0}}^{\substack{j=2 g+2 \\ i=2 g+2}} a_{i} s_{i-\left\lceil\frac{j}{2}\right\rceil} s_{m+\left\lceil\frac{j}{2}\right\rceil}+\sum_{\substack{i=0 \\ j=1}}^{\substack{j=m-1 \\ i=m-j-1}} a_{i} s_{i+\left\lfloor\frac{j}{2}\right\rfloor} s_{m-\left\lfloor\frac{j}{2}\right\rfloor}+\sum_{i=0}^{2 g+2} a_{i} s_{\left\lceil\frac{m+i}{2}\right\rceil} s_{\left\lfloor\frac{m+i}{2}\right\rfloor} .
$$


In particular, if we take the curve $y^{2}-1+x^{2 g+2}$, we have:

$$
t s_{m}+\sum_{j=1}^{2 g+1-m} s_{2 g+2-\left\lceil\frac{j}{2}\right\rceil} s_{m+\left\lceil\frac{j}{2}\right\rceil}+\sum_{j=2}^{m} s_{\left\lfloor\frac{j}{2}\right\rfloor} s_{m-\left\lfloor\frac{j}{2}\right\rfloor}, \quad m=1, \ldots, 2 g+1 .
$$

For $g=2$ there are five equations: $t s_{1}+2 s_{5} s_{2}+2 s_{4} s_{3}, t s_{2}+2 s_{5} s_{3}+s_{4}^{2}+s_{1}^{2}$, $t s_{3}+2 s_{5} s_{4}+2 s_{1} s_{2}, t s_{4}+s_{5}^{2}+2 s_{1} s_{3}+s_{2}^{2}, t s_{5}+2 s_{1} s_{4}+2 s_{2} s_{3}$. This is a complete intersection of degree $2^{5}$ : it is the cone over 32 distinct points.

We shall prove in the next Section that the base space is always a complete intersection. It is difficult to see this from the above equations.

\section{Smoothing components}

(3.1) In this section we prove that the cone over a hyperelliptic curve of degree $4 g+4$ has $2^{2 g+1}$ smoothing components. To each component corresponds a surface with the curve as hyperplane section; these are ruled surfaces, and we show how to obtain all by elementary transformations on a given one. To this end we identify the one dimensional subspace in $T^{1}$, determined by the surface.

Let $\bar{X} \subset \mathbb{P}^{N+1}$ be the projective cone over a projectively normal algebraic variety $C \subset \mathbb{P}^{N}$ of dimension $n$, and let $S \subset \mathbb{P}^{N+1}$ be variety of dimension $n+1$ with $C$ as hyperplane section. Then there exists a one parameter deformation of $\bar{X}$ with $S$ as general fibre: let $\bar{Y} \subset \mathbb{P}^{N+2}$ be the projective cone over $S$, and consider the pencil $\left\{H_{t}\right\}$ of hyperplanes through $\mathbb{P}^{N} \supset C$; let the hyperplane $H_{0}$ pass through the vertex, then $\bar{Y} \cap H_{0}=\bar{X}$, while for $t \neq 0$ the projection from the vertex establishes an isomorphism between $\bar{Y} \cap H_{t}$ and $S$. Pinkham calls this construction 'sweeping out the cone' [Pinkham 1970]; the degeneration to the cone occurs already in the famous 'Anhang F' [Severi 1921].

For the affine cone $X$ over $C$ we have a deformation with Milnor fibre $S-C$. The versal base $S_{X}^{-}$in negative degree is a fine moduli space for so called $R$ polarised schemes [Looijenga 1984], a notion defined in general for quasi-homogeneous spaces; in our situation this are spaces $S$ with $C$ as hyperplane section. Basically one considers the coordinate $t$, which defines the hyperplane section, as deformation parameter.

Let $S$ be given by equations $F_{i}(\boldsymbol{x}, t)=f_{i}(\boldsymbol{x})+t f_{i}^{(1)}+\ldots+t^{d_{i}} f_{i}^{\left(d_{i}\right)}$, in homogeneous coordinates $(\boldsymbol{x}, t)$, where $\operatorname{deg} f_{i}^{(j)}=d_{i}-j$. The base space of $X$ has a $\mathbb{C}^{*}$-action. For simplicity we assume that the only occurring negative degree is -1 , as in our application. The by $S$ induced infinitesimal deformation of $X$ is given by $\left.f_{i} \mapsto \frac{\partial}{\partial t} F_{i}(\boldsymbol{x}, t)\right|_{t=0}=f_{i}^{(1)}$. This can be interpreted as section of $H^{0}\left(C, N_{C}(-1)\right)$.

Let $S$ be embedded by the line bundle $L$, and let $\mathbb{P}=\mathbb{P}\left(H^{0}(S, L)^{*}\right)$. Let $C=H \cap S$, with $H$ a hyperplane in $\mathbb{P}$, and let $i: C \rightarrow S$ be the inclusion. Then $N_{C / S}=L_{\left.\right|_{C}}$. In the exact sequence

$$
0 \longrightarrow \mathcal{O}_{C} \cong N_{C / S}(-1) \longrightarrow N_{C / \mathbb{P}}(-1) \longrightarrow i^{*} N_{S / \mathbb{P}}(-1) \longrightarrow 0
$$

the infinitesimal deformation is the image of $1 \in \mathcal{O}_{C}$. The curve $C$ is minimally embedded in the hyperplane $H$, so we really want a section of $N_{C / H}(-1)$; the 
exact sequence

$$
0 \longrightarrow N_{C / H}(-1) \longrightarrow N_{C / \mathbb{P}}(-1) \longrightarrow i^{*} N_{H / \mathbb{P}}(-1) \longrightarrow 0
$$

splits: $N_{H / \mathbb{P}}(-1)$ is generated by the global section, which sends the equation $t$ to 1 , and this section can be mapped to the section $\left.F \mapsto \frac{\partial}{\partial t} F\right|_{t=0}$ of $N_{C / \mathbb{P}}(-1)$.

For curves we have a third description, which uses $\left(T^{1}\right)^{*}$. We recall from Proposition (1.3) the sequence $0 \longrightarrow N_{C}^{*} \otimes L \longrightarrow V \otimes_{\mathbb{C}} \mathcal{O}_{C} \longrightarrow \mathcal{P}_{C}^{1}(L) \longrightarrow 0$. We have seen that $T_{X}^{1}(-1)^{*}=\operatorname{coker}\left\{V \otimes H^{0}(K) \rightarrow H^{0}\left(\mathcal{P}_{C}^{1}(L) \otimes K\right)\right\}$. Consider the following diagram of exact sequences:

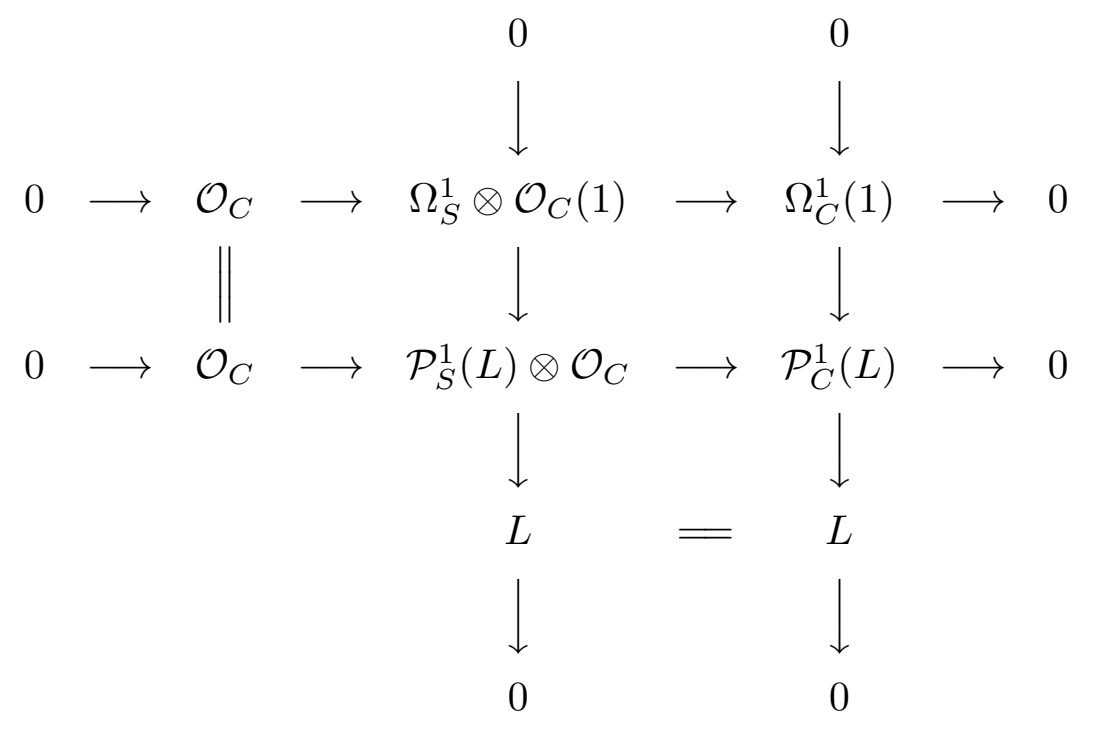

(3.2) Proposition. Let $\xi \in H^{0}\left(C, N_{C}(-1)\right)$ be the by $S$ induced infinitesimal deformation of the cone over $C$. The map $\cup \xi: T^{1}(-1)^{*} \rightarrow \mathbb{C}$ is the connecting homomorphism $H^{0}\left(\mathcal{P}_{C}^{1}(L) \otimes K\right) \rightarrow H^{1}(K)$, obtained from the above sequence by tensoring with $K=\Omega_{C}^{1}$; alternatively one may consider the map $H^{0}\left(K^{2} L\right) \rightarrow$ $H^{1}(K)$.

Proof. We first describe $H^{0}\left(\mathcal{P}^{1}(L) \otimes K\right)$. The sheaf $\mathcal{P}^{1}(L)$ is generated by global sections; if we take a local coordinate $u$ on $C$ and write a Newton dot for the derivative with respect to $u$, then the map $V \otimes \mathcal{O}_{C} \rightarrow \mathcal{P}^{1}(L)$ is given in accordance with our earlier notations by $d z_{i} \mapsto z_{i}(u)+\dot{z}_{i}(u) d u$. Let $\phi$ be a global section of $\mathcal{P}^{1}(L) \otimes K$. On $U_{j}=\left\{z_{j} \neq 0\right\}$ the section $\phi$ can be represented by $\sum \omega_{j}^{i} d z_{i}$, and the representations for different $j$ are connected by the conditions $\sum \omega_{j}^{i} z_{i}=\sum \omega_{k}^{i} z_{i}$ and $\sum \omega_{j}^{i} \dot{z}_{i}=\sum \omega_{k}^{i} \dot{z}_{i}$

This allows us to compute $\phi \cup \xi$ : the cochain $\sum\left(\omega_{k}^{i}-\omega_{j}^{i}\right) d z_{i}$ represents an element of $H^{1}\left(N_{C}^{*}(1) \otimes \Omega_{C}^{1}\right)=H^{0}\left(N_{C}(-1)\right)^{*}$. To express $\xi$ in terms of the $\frac{\partial}{\partial z_{i}}$, we take local coordinates $(u, \tau)$ on $S$ with $\tau$ vanishing on $C$, and we denote differentation w.r.t. $\tau$ by ' . For every equation $F$ of $S$ we have $\partial F / \partial \tau=0$, so:

$$
t^{\prime}(u, 0) \frac{\partial F}{\partial t}+\sum z_{i}^{\prime}(u, 0) \frac{\partial F}{\partial z_{i}}=0
$$


and therefore $\xi=-\sum\left(z_{i}^{\prime} / t^{\prime}\right) \frac{\partial}{\partial z_{i}}$. Now $\phi \cup \xi=\sum\left(\omega_{j}^{i}-\omega_{k}^{i}\right)\left(z_{i}^{\prime} / t^{\prime}\right)$.

To compute the connecting homomorphism $\delta$ in the exact sequence:

$$
0 \rightarrow \Omega_{C}^{1} \rightarrow \mathcal{P}_{S}^{1}(L) \otimes \Omega_{C}^{1} \rightarrow \mathcal{P}_{C}^{1}(L) \otimes \Omega_{C}^{1} \rightarrow 0,
$$

we lift $\phi$ on $U_{j}$ to $\mathcal{P}^{1}(L) \otimes K$. On $U_{j}=\left\{z_{j} \neq 0\right\}$ we write $\phi$ as $\sum \omega_{j}^{i} d z_{i}+\omega_{j} d t$, and the cochain conditions on $S$ are: $\sum \omega_{j}^{i} z_{i}+\omega_{j} t=\sum \omega_{k}^{i} z_{i}+\omega_{k} t, \sum \omega_{j}^{i} \dot{z}_{i}+\omega_{j} \dot{t}=$ $\sum \omega_{k}^{i} \dot{z}_{i}+\omega_{k} \dot{t}$ and $\sum \omega_{j}^{i} z_{i}^{\prime}+\omega_{j} t^{\prime}=\sum \omega_{k}^{i} z_{i}^{\prime}+\omega_{k} t^{\prime}$; for $\tau=0$ we have $t=\dot{t}=0$, so the first two conditions are the same as before, and we have $\left(\omega_{k}-\omega_{j}\right) t^{\prime}=\sum\left(\omega_{j}^{i}-\omega_{k}^{i}\right) z_{i}^{\prime}$. The cochain $\omega_{k}-\omega_{j}$ represents $\delta(\phi) \in H^{1}(K)$, and therefore $\delta(\phi)=\phi \cup \xi$.

We now concentrate on the hyperelliptic case. The cone over a hypereliptic curve of degree $d \leq 4 g+4$ is smoothable. More precisely, we have:

(3.3) Proposition [Tendian 1992b]. Let ${ }^{g} C$ be a hyperelliptic curve, embedded with a complete linear system $L$ of degree $7 g / 3+1 \leq d \leq 4 g+4$. Then $C$ is a hyperplane section of a projectively normal surface $\bar{S}=\phi_{|C|}(S)$, where $S$ can be obtained from a rational ruled surface by blowing up $4 g+4-d$ points. The dimension of the smoothing component is $7 g+4-d+h^{0}\left(C, K^{2} L^{-1}\right)$. If $d>\max \{4 g-4,3 g+6\}$ for $g \neq 6$, and $d>25$ for $g=6$, then every smoothing component is of this form, and has dimension $7 g+4-d$.

In particular, for $d=4 g+4$ the surface $S$ is ruled, and the dimension of the smoothing component is $3 g=(2 g-1)+g+1$. The dimension of the hyperelliptic locus is $2 g-1$, and $g$ is the dimension of $\operatorname{Pic}^{d}$. So for general $(C, L)$ each smoothing component determines a unique surface $\bar{S}$ with $C$ as hyperplane section. Furthermore $\operatorname{dim} T_{X}^{1}(-1)=2 g+2$, and the base space in negative degree is given by $2 g+1$ equations. From these facts we cannot yet conclude that the equations define a complete intersection.

The existence of smoothings can be shown in the following way: given a line bundle $L$ of degree $4 g+4$ on $C$ (which is not necessarily the linear system for which we want a smoothing — we denote that temporarily by $N$ ), we consider the scroll $\bar{S}$ of type $(a, b)$, on which $\phi_{L}(C)$ lies: it is the image of the ruled surface $S=\mathbb{P}\left(\pi_{*} L\right)$, cf. (2.2). On $S$ the hyperplane class is $L=E_{0}+a f$, and the curve $C$ is a divisor of type $2 E_{0}+(g+1+e) f=2 L-(2 g+2) f$; here $e=a-b$. So for every $L$ with $L^{2} \cong N \otimes\left(g_{2}^{1}\right)^{2 g+2}$ the normal bundle $N_{C / S}$ is isomorphic to our fixed bundle $N$. The number of solutions to this equation is the order of the group $J_{2}(C)$ of 2-torsion points on $\operatorname{Jac}(C)$, which is $2^{2 g}$.

For all surfaces $S$ obtained by this construction we have $e \equiv g+1 \quad(\bmod 2)$, because $e=a-b$, and $a+b=3 g+3$. This is not surprising, because for a fixed curve and variable $L$ the surfaces $\mathbb{P}\left(\pi_{*} L\right)$ form a continuous family, and the parity of $e$ is conservated under deformations.

Let $C$ lie on on the ruled surface $S \cong F_{e}$, with normal bundle $N_{C / S}=N$, with $0 \leq e \leq g+1$. Denote the elementary transformation [Hartshorne 1977, V.5.1.7] of $S$ in $Q \in S$ by $\operatorname{elm}_{Q}$. Then we have the following simple, but important observation:

(3.4) Lemma. Let $C^{\prime}$ be the strict transform of $C$ on $S^{\prime}=\operatorname{elm}_{P}(S)$, where $P$ is a Weierstraß point. Then the normal bundle $N_{C^{\prime}} / S^{\prime}$ is equal to the normal bundle $N_{C / S}$. 
This lemma shows the existence of ruled surfaces $C \subset S \cong F_{e}$ with $N_{C / S}=N$ and $g+1+e$ odd; again $C$ is of type $2 E_{0}+(g+1+e) f$.

(3.5) Proposition. Let $B$ be the set of Weierstraß points on $C$. Let $\operatorname{elm}_{B}$ be the composition of the elementary transformations in all $P \in B$. Then $\operatorname{elm}_{B}(S)$ is isomorphic to $S$, under an isomorphism $I$, which leaves $C$ pointwise fixed; on the general fibre $f$ of $S \rightarrow \mathbb{P}^{1}$, I restricts to the unique involution with $C \cap f$ as fixed points. The isomorphims $I$ maps the linear system $|C|$ on $S$ to the linear system $\left|C^{\prime}\right|$ on $S^{\prime}$.

Proof. The rational map $\operatorname{elm}_{B}$ can be factorised as $S \stackrel{\sigma}{\longleftarrow} \widetilde{S} \stackrel{\sigma^{\prime}}{\longrightarrow} S^{\prime}$, where the maps $\sigma$ and $\sigma^{\prime}$ are the blowups in the points of $B$, with exceptional curves $E_{i}$ and $E_{i}^{\prime}$. The involution on the general fibre fof $\widetilde{S} \rightarrow \mathbb{P}^{1}$ with $f \cap C$ as fixed points extends to an involution $\widetilde{I}$ on $\widetilde{S}$, which interchanges $E_{i}$ and $E_{i}^{\prime}$. This map descends to the required isomorphism. The inverse image on $\widetilde{S}$ of the linear system $|C|$ is $\left|\bar{C}+\sum E_{i}\right|$, where $\bar{C}$ is the strict transform of $C$. The involution $\widetilde{I}$ transforms this system into $\left|\bar{C}+\sum E_{i}^{\prime}\right|=\left(\sigma^{\prime}\right)^{*}\left|C^{\prime}\right|$.

(3.6) Proposition. Let $C \subset S \cong F_{e}$ be a curve of type $2 E_{0}+(g+1+e) f$ with normal bundle $N_{C / S}=N$. Denote for a subset $T \subset B$ by $\operatorname{elm}_{T}$ the composition of the $\operatorname{elm}_{P_{i}}, P_{i} \in T$. Two surfaces $\operatorname{elm}_{T_{1}}(S)$ and $\operatorname{elm}_{T_{2}}(S)$ induce the same deformation of the cone $X(C, N)$ if and only if $T_{1}=T_{2}$ or $T_{1}=B \backslash T_{2}$.

Proof. We have to compute the connecting homomorphism from the exact sequence:

$$
0 \rightarrow \Omega_{C}^{1} \rightarrow \Omega_{S}^{1} \otimes \Omega_{C}^{1}(1) \rightarrow\left(\Omega_{C}^{1}\right)^{\otimes 2}(1) \rightarrow 0,
$$

or, what amounts to the same, the hyperplane in $H^{0}\left(C, K^{2}(1)\right)$, which is the image of $H^{0}\left(C, \Omega_{S}^{1} \otimes \Omega_{C}^{1}(1)\right)$. We have on $S$ the sequence:

$$
0 \rightarrow \pi^{*} \Omega_{\mathbb{P}^{1}}^{1} \rightarrow \Omega_{S}^{1} \rightarrow \Omega_{S / \mathbb{P}^{1}}^{1} \rightarrow 0
$$

and on $C$ :

$$
0 \rightarrow \pi^{*} \Omega_{\mathbb{P}^{1}}^{1} \rightarrow \Omega_{C}^{1} \rightarrow \Omega_{C / \mathbb{P}^{1}}^{1} \rightarrow 0
$$

where $\Omega_{C / \mathbb{P}^{1}}^{1}=T_{X}^{1}(-1)^{*} \cong \oplus_{P \in B} \mathbb{C}_{P}$. The map $\Omega_{S}^{1} \otimes \Omega_{C}^{1}(1) \rightarrow\left(\Omega_{C}^{1}\right)^{\otimes 2}(1)$ is an isomorphism on the subspace $\pi^{*} \Omega_{\mathbb{P}^{1}}^{1} \otimes \Omega_{C}^{1}(1)$. Because $K_{S}=-2 E_{0}-(e+2) f$, we have $\Omega_{S / \mathbb{P}^{1}}^{1}=\mathcal{O}_{S}\left(-2 E_{0}-e f\right)$, and $\Omega_{S / \mathbb{P}^{1}}^{1}(C+(g-1) f)=\mathcal{O}_{S}(2 g f)$. We identify $T_{X}^{1}(-1)^{*}$ with $H^{0}\left(C,\left(\Omega_{C}^{1} / \pi^{*} \Omega_{\mathbb{P}^{1}}\right) \otimes \Omega_{C}^{1}(1)\right)$ by taking in each Weierstraß point $P_{i}$ a generator of $K^{2}(1) / \mathfrak{m}_{P_{i}} K^{2}(1)$; the map from $H^{0}\left(C, \Omega_{S / \mathbb{P}^{1}}^{1} \otimes \Omega_{C}^{1}(1)\right)$ consists in taking the coefficients in the points $P_{i}$. The sections of $\left(g_{2}^{1}\right)^{\otimes 2 g}$ are $1, \ldots, x^{2 g}$, which come from sections on $S$, and $y, \ldots, y x^{g-1}$, which vanish in the Weierstraß points. Therefore, if we take coordinates $s_{i}$ on $T_{X}^{1}(-1)^{*}$, and if $x$-coordinate of $P_{i}$ is $a_{i}$, then the image of $H^{0}\left(C, \Omega_{S / \mathbb{P}^{1}}^{1} \otimes \Omega_{C}^{1}(1)\right)$ is given by the determinant:

$$
D\left(s_{1}, \ldots, s_{2 g+2}\right)=\left|\begin{array}{cccccc}
1 & a_{1} & a_{1}^{2} & \ldots & a_{1}^{2 g} & s_{1} \\
\vdots & \vdots & \vdots & \ddots & \vdots & \vdots \\
1 & a_{2 g+2} & a_{2 g+2}^{2} & \ldots & a_{2 g+2}^{2 g} & s_{2 g+2}
\end{array}\right| .
$$


Now we consider the surface $S^{\prime}=\operatorname{elm}_{T}(S)$ for some $T \subset B$. Again we can identify the sections of $H^{0}\left(C, \Omega_{S^{\prime} / \mathbb{P}^{1}}^{1} \otimes \Omega_{C}^{1}(1)\right)$, coming from $S^{\prime}$, with the polynomials 1 , $\ldots, x^{2 g}$, but we have to express these in the same basis of $T_{X}^{1}(-1)^{*}$, which we used for $S$. Consider local coordinates $(x, y)$ in a neighbourhood of a point $P \in T$, such that ruling is given by $\pi(x, y)=x$, and $C$ is $y^{2}=x$. We blow $S$ up in $P$; the strict transform of $C$ passes through the origin of the $(\eta, y)$ coordinate patch, where $(x, y)=(\eta y, y)$. Now blow down the $y$-axis: we have coordinates $(\xi, \eta)=(\eta y, \eta)$, so $x=\xi, y=\xi / \eta$. Therefore the local generator $d y$ of $\Omega_{C, P}^{1}$ is transformed into $d \eta$ : we have $d y=d \xi / \eta-\xi d \eta / \eta^{2}$; however, considered as section of $\Omega_{S}^{1} / \pi^{*} \Omega_{\mathbb{P} 1}^{1}$, the formula makes sense for $\eta \neq 0$ and is on $C$ the same as $-\xi d \eta / \eta^{2}=-d \eta$. This computation shows that $S^{\prime}$ yields the hyperplane:

$$
D\left((-1)^{\chi_{T}\left(P_{1}\right)} s_{1}, \ldots,(-1)^{\chi_{T}\left(P_{2 g+2}\right)} s_{2 g+2}\right)=0
$$

where $\chi_{T}$ is the characteristic function of $T$, i.e $\chi_{T}\left(P_{i}\right)=1$ if and only if $P_{i} \in T$.

To finish the proof we remark that the coefficient of $s_{i}$ in the equation $D$ is a Vandermonde determinant, and therefore non zero.

(3.7) Theorem. Let $X$ be the cone over a hyperelliptic curve $C$ of genus $g$, embedded with a complete linear system $L$ of degree $4 g+4$. Suppose $L \neq 4 K$, if $g=3$. Then $X$ has $2^{2 g+1}$ smoothing components.

Proof. The number of subsets $T$ of $B$ modulo the equivalence relation $T \sim B \backslash T$ is $2^{2 g+1}$. So Proposition (3.6) gives this number of one parameter smoothings of $X$. After a rescaling these define in $\mathbb{P}\left(T_{X}^{1}(-1)\right)$ the points $( \pm 1: \ldots: \pm 1)$, and this is a complete intersection. In coordinates $s_{i}$ on $\mathbb{P}\left(T_{X}^{1}(-1)\right)$ the ideal of these points is generated by $s_{i}^{2}-s_{j}^{2}$. The $2 g+1$ quadratic equations for the base in negative degree are contained in this ideal; they generate this ideal if and only if they are linearly independent. Because the dimension of each smoothing component is $3 g$, the equations are independent for generic $(C, L)$. Suppose that for some special $(C, L)$ the equations are dependent. Then the base space of $X(C, L)$ is not smooth along at least one of the parameter lines of the $2^{2 g+1}$ smoothing we just constructed. But the only singularities in the fibres are cones over a rational normal curve of degree $g+1$, which have a smooth reduced base space, if $g \neq 3$. Therefore the equations are always independent.

For $g=3$ we still have the same description of $S^{-}$, but in case $L=4 K$ the cone over the rational normal curve of degree 4 appears as singularity over one component. Its Veronese smoothing leads to an additional smoothing component; it exists also for the non hyperelliptic curves.

(3.8) Remark. The set $\mathcal{B}$ of subsets $T$ of $B$ modulo the equivalence relation $T \sim B \backslash T$ forms a group, isomorphic to $\mathbb{Z}^{2 g+1}$; the subgroup $\mathcal{B}^{+}$of $T$ 's of even cardinality is isomorphic to $J_{2}(C)$ : every $\eta \in J_{2}(C)$ can be represented by a divisor $D \sim k g_{2}^{1}-\sum_{i \in T} P_{i}$, with $|T|=2 k$.

(3.9) Lemma. Let $\eta \in J_{2}(C)$ be represented by $D \sim k g_{2}^{1}-\sum_{i \in T} P_{i}$, with $T \in \mathcal{B}^{+}$. Let $S=\mathbb{P}\left(\pi_{*} L\right)$. Then $S_{\eta}=\mathbb{P}\left(\pi_{*}(L \otimes \eta)\right)$ is isomorphic to $\operatorname{elm}_{T}(S)$. 
Proof. Factorise $\operatorname{elm}_{T}$ as $S \stackrel{\sigma}{\longleftarrow} \widetilde{S} \stackrel{\sigma^{\prime}}{\longrightarrow} S^{\prime}=\operatorname{elm}_{T}(S)$ : as before, the exceptional divisors are $E_{i}$ and $E_{i}^{\prime}$. We have $L=E_{0}+a f$; let $T_{0} \subset T$ be the index set of points $P_{i}$ on $E_{0}$, with cardinality $m$. Let $\bar{E}_{0}$ be the strict transform of $E_{0}$ on $\widetilde{S}$, and $E_{0}^{\prime}$ that on $S^{\prime}$. Then:

$$
\begin{aligned}
\sigma^{*} L & =\bar{E}_{0}+\sum_{j \in T_{0}} E_{j}+a f \\
& =\left(\sigma^{\prime}\right)^{*} E_{0}^{\prime}-\sum_{i \notin T_{0}} E_{i}^{\prime}+\sum_{j \in T_{0}} E_{j}+a f \\
& \sim\left(\left(\sigma^{\prime}\right)^{*} E_{0}^{\prime}+(a+m-k) f\right)+\sum_{T} E_{i}-k f .
\end{aligned}
$$

On $S^{\prime}$ the linear system $\left|E_{0}^{\prime}+(a+m-k) f\right|$ cuts out on $C$ the series $|L+D|$. We have $E_{0}^{\prime} \cdot E_{0}^{\prime}=-e+2(k-m)$, so if $2(k-m)<e$, the section $E_{0}^{\prime}$ is the unique negative section, and we see directly that $E_{0}^{\prime}+(a+m-k) f$ is the hyperplane class $(2 a=3 g+3+e)$. Otherwise there is a section $E_{-}$with $E_{-} \cdot E_{-}=-e^{\prime}, e^{\prime} \geq 0$, and $E_{0}^{\prime} \sim E_{-}+\left(k-m-e / 2+e^{\prime} / 2\right) f$. The hyperplane class is $E_{-}+\left(3 g+3+e^{\prime}\right) / 2 f \sim$ $E_{0}^{\prime}+(a+m-k) f$.

(3.10) Alternative description of the construction. We have given the surfaces $S$ as ruled surfaces, birationally embedded with the linear system $|C|=\left|2 E_{0}+a f\right|$. Castelnuovo decribes linear systems in the plane, of curves of degree $g+e+1$ with one $(g+e-1)$-ple point and $e-1$ infinitely near double points, if $1 \leq e \leq g+1$, or for $e=0$ of curves of degree $g+3$ with a $(g+1)$-ple point and a double point in a different point [Castelnuovo 1890]. If there are at least two finite double points, a standard Cremona transformation will decrease the degree, for details see the book [Conforto 1939].

Now given a curve $C$ in the plane of degree $d$ with $\delta$ double points (finite or not), and with multiplicity $d-2$ at the origin, and given a set $T$ of Weierstraß points of cardinality $k$, we form the curve $C \cup\left(\cup_{i \in T} L_{i}\right)$ of degree $d+k$, where $L_{i}$ is the line joining the origin with the Weierstraß point $P_{i}$, and we consider the linear system of plane curves of degree $d+k$ with multiplicity $d+k-2$ at the origin, and $\delta+k$ double points in the double points of $C$ and in the $P_{i}, i \in T$. As the curves of this system do not intersect $L_{i}$ outside $C$, the rational map $\phi_{|C|}\left(\mathbb{P}^{2}\right) \rightarrow \phi_{\left|C+\sum L_{i}\right|}\left(\mathbb{P}^{2}\right)$ is $\operatorname{elm}_{T}$.

(3.11) Example ((2.13) continued). Let $X$ be the cone over the hyperelliptic curve $y^{2}-1+x^{6}$, embedded with $6 g_{2}^{1}$. The coordinates on $T_{X}^{1}(-1)$ used in $(2.13)$ are different from the ones in the proof of Proposition (3.6); in principle one can compute the coordinate transformation, but we will not do this here. We describe two surfaces with $C$ as hyperplane section, and identify the induced line in $T_{X}^{1}(-1)$. We remark that the Weierstraß points are the sixth roots of unity, and that the group $\mu_{6}$ operates on $\mathcal{B}$.

We start with the plane curve $x^{2} y^{2} z^{2}-y^{6}+x^{6}$. The curves of degree 6 with multiplicity 4 in $O$, and $A_{3}$ singularities, tangent to $x=0$ and $y=0$, are:

$$
z_{i}=y^{6-i} x^{i}, \quad i=0, \ldots, 6 ; \quad w_{i}=z y x y^{3-i} x^{i}, \quad i=0, \ldots, 3 ; \quad u=z^{2} y^{2} x^{2} .
$$


This gives equations:

$$
\begin{gathered}
\operatorname{Rank}\left(\begin{array}{cccccc}
z_{0} & \ldots & z_{5} & w_{0} & w_{1} & w_{2} \\
z_{1} & \ldots & z_{6} & w_{1} & w_{2} & w_{3}
\end{array}\right) \leq 1, \\
\phi_{m}=w_{\left\lfloor\frac{m}{2}\right\rfloor} w_{\left\lceil\frac{m}{2}\right\rceil}-u z_{m}, \quad m=0, \ldots, 6 .
\end{gathered}
$$

The image of $\mathbb{P}^{2}$ is isomorphic to the cone over the rational normal curve of degree 3. With $t=u-z_{0}+z_{6}$ we get a deformation of $C$, with $s_{i}=0$.

Now consider the Weierstraß point $P_{0}=(1: 1: 0)$, and the linear system $\left|C+L_{0}\right|$ of septic curves with additional double point at $P_{0}$. A basis is given by:

$$
\begin{gathered}
\zeta_{i}=(x-y)^{2} y^{5-i} x^{i}, \quad i=0, \ldots, 5 ; \quad u_{0}=z^{2} y^{3} x^{2}, \quad u_{1}=z^{2} y^{2} x^{3} \\
w_{i}=z y x(x-y) y^{3-i} x^{i}, \quad i=0, \ldots, 3 .
\end{gathered}
$$

On $C$ we have $(y-x)^{-1} \zeta_{i}=z_{i}-z_{i+1}$, and $(y-x)^{-1} u_{0}=z_{0}+\ldots+z_{5},(y-x)^{-1} u_{1}=$ $z_{1}+\ldots+z_{6}$; therefore we find as equalities on $C$ :

$$
\begin{aligned}
& 6(y-x) z_{0}=u_{0}+5 \zeta_{0}+4 \zeta_{1}+3 \zeta_{2}+2 \zeta_{3}+\zeta_{4} \\
& 6(y-x) z_{1}=u_{0}-\zeta_{0}+4 \zeta_{1}+3 \zeta_{2}+2 \zeta_{3}+\zeta_{4} \\
& 6(y-x) z_{2}=u_{0}-\zeta_{0}-2 \zeta_{1}+3 \zeta_{2}+2 \zeta_{3}+\zeta_{4} \\
& 6(y-x) z_{3}=u_{0}-\zeta_{0}-2 \zeta_{1}-3 \zeta_{2}+2 \zeta_{3}+\zeta_{4} \\
& 6(y-x) z_{4}=u_{0}-\zeta_{0}-2 \zeta_{1}-3 \zeta_{2}-4 \zeta_{3}+\zeta_{4} \\
& 6(y-x) z_{5}=u_{0}-\zeta_{0}-2 \zeta_{1}-3 \zeta_{2}-4 \zeta_{3}-5 \zeta_{4} \\
& 6(y-x) z_{6}=u_{0}-\zeta_{0}-2 \zeta_{1}-3 \zeta_{2}-4 \zeta_{3}-5 \zeta_{4}-6 \zeta_{5} .
\end{aligned}
$$

Furthermore, $C$ is given by $u_{0}-u_{1}-\sum \zeta_{i}=\tau$. The matrix for the $\operatorname{scroll}_{\ln }(S)$ has as first entry $6(y-x) z_{0}$; i.e. we make a coordinate change $\rho z_{0}:=u_{0}+5 \zeta_{0}+4 \zeta_{1}+$ $3 \zeta_{2}+2 \zeta_{3}+\zeta_{4}$ (we are allowed to multiply homogeneous coordinates with a common factor $)$. Below $6(y-x) z_{0}$ we have to write $6(y-x) z_{1}=u_{1}+5 \zeta_{1}+4 \zeta_{2}+3 \zeta_{3}+2 \zeta_{4}+\zeta_{5}$; the second entry on the first row is $u_{0}-\zeta_{0}+4 \zeta_{1}+3 \zeta_{2}+2 \zeta_{3}+\zeta_{4}=6(y-x) z_{1}+\tau$. Therefore $s_{1}=\tau$; proceeding in this way we find $s_{i}=\tau$ for $i=1, \ldots, 5$. For the coordinates $w_{i}$ we take $6(y-x) z y x y^{3-i} x^{i}$. Finally we have to compute $t$ in terms of $\tau$. This is now a simple question of making the explicitly given coordinate transformation in the equations, and the best way to do this is by computer. One finds $t=-4 \tau$. Indeed, $s_{i}=\tau$ and $t=-4 \tau$ is a solution of the equations for the base space.

The other components are found with similar computations.

(3.12) The Milnor fibre. In their paper [Looijenga-Wahl 1986] Looijenga and Wahl introduce a collection of smoothing data for singularities, which in many cases distinguish between components. We determine these data in our case.

Because the cone over an hyperelliptic curve $C$ of degree $4 g+4$, is a homogeneous singularity, the interior of the Milnor fibre of a one parameter smoothing, given by a surface $S$ with $C$ as hyperplane section, is isomorphic to the open surface $S \backslash C$. We have described all smoothing components by smooth ruled surfaces, 
except when $\bar{S}$ is isomorphic to the cone over a rational normal curve of degree $g+1$; then $N_{C / S}=(2 g+2) g_{2}^{1}$. In the last case there does not exist a one-parameter smoothing, which lies totally in $S^{-}$, but by slightly changing the normal bundle we find a smooth ruled surface $S$ on the same smoothing component, and the Milnor fibre is again $S \backslash C$.

(3.13) Lemma. Let $C$ be a curve of type $2 E_{0}+(g+1+e) f$ on $S \cong F_{e}$. Then the Milnor fibre $F$ of the cone $X$ over $C$ has homology $H_{2}(F, \mathbb{Z})=\mathbb{Z}^{2 g+1}$ and $H_{1}(F)=\mathbb{Z} / 2$. If $g+e+1 \cong 0 \quad(\bmod 2)$, then $H_{1}(F)=\mathbb{Z} / 2$ and $H^{2}(F, \partial F)=$ $\mathbb{Z}^{2 g+1} \oplus \mathbb{Z} / 2$; otherwise $H_{1}(F)=0$ and $H^{2}(F, \partial F)=\mathbb{Z}^{2 g+1}$.

Proof. We compute the homology of $F=S \backslash C$ with the exact homology sequence of the pair $(S, S \backslash C)$. For the surface $S$ we have $H^{3}(S)=H^{1}(S)=0$ and $H_{2}(S)=$ $\operatorname{Pic}(S)$. We may take a new basis, consisting of $E=E_{0}+\left\lfloor\frac{g+1+e}{2}\right\rfloor f$ and $f$. Then $C \sim 2 E+\epsilon f$ with $\epsilon=0$ if $g+1+e$ is even, and 1 otherwise, and $E \cdot E=g+1-\epsilon$. We have already encountered this division in cases. Let $N$ be a tubular neighbourhood of $C$; by excision and Poincaré Duality $H_{i}(S, S \backslash C)=H_{i}(N, \partial N) \cong H^{4-i}(C)$. Therefore we have:

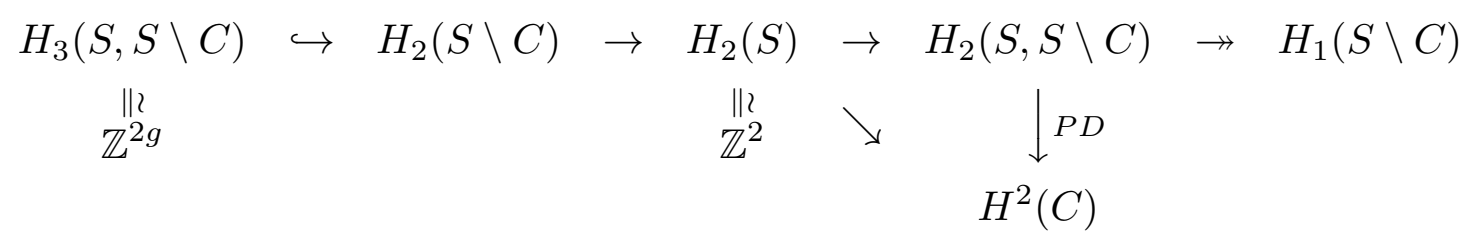

The composed map $H_{2}(S) \rightarrow H^{2}(C)$ is given by intersecting with the curve $C$; so $f \mapsto 2$ and $E \mapsto 2 g+2-\epsilon$. If $\epsilon=0$, then $H_{1}(F ; \mathbb{Z})=\mathbb{Z} / 2$, and $H_{1}(F ; \mathbb{Z})=0$, if $\epsilon=1$. In the odd case $\operatorname{ker}\left\{H_{2}(S) \rightarrow H_{2}(S, S \backslash C)\right\}$ is generated by $2 E-(2 g+2-\epsilon) f$, and $(2 E-(2 g+2-\epsilon) f)^{2}=-4(g+1)$; in the even case a generator is $E-(g+1) f$, with self-intersection $-(g+1)$.

We compute $H_{2}(F, \partial F) \cong H^{2}(F)$ with the cohomology sequence of the pair $(S, S \backslash C)$. We obtain:

$$
0 \longrightarrow H^{2}(S, S \backslash C) \longrightarrow H^{2}(S) \longrightarrow H^{2}(S \backslash C) \longrightarrow H^{3}(S, S \backslash C) \longrightarrow 0 .
$$

By Poincare duality $H^{i}(S, S \backslash C ; \mathbb{Z}) \cong H_{4-i}(C)$, and the map $H_{2}(C) \rightarrow H^{2}(S)$ is the transpose of the map $H_{2}(S) \rightarrow H^{2}(C)$ above.

The boundary $\partial F$ of the Milnor fibre is diffeomorphic to the link $M$ of the singularity. As $M$ is a circle bundle over $C$, we have that $H_{1}(M)=\mathbb{Z}^{2 g} \oplus \mathbb{Z} /(4 g+4)$. The linking form on $H_{1}(M)_{t}$, the torsion part, lifts to a quadratic function, which can be computed from the resolution [Looijenga-Wahl 1986, 4.2, 4.6]. We have, with $\bar{e}$ a generator of $H_{1}(M)_{t}$ :

$$
q: H_{1}(M)_{t} \rightarrow \mathbb{Q} / \mathbb{Z}, \quad q(m \bar{e})=\frac{m(m-6 g-2)}{8 g+8} .
$$

By [loc. cit., Thm. 4.5] the group $H_{2}(F)$, with intersection form $\mathcal{I}$, carries the structure of a quadratic lattice with associated non-degenerate lattice $\bar{H}_{2}(F)=$ 
$H_{2}(F) / \operatorname{rad} \mathcal{I}$, whose discriminant quadratic function is canonically isomorphic to $\left(I^{\perp} / I, q_{I}\right)$, where $I$ is the $q$-isotropic subgroup $\operatorname{Im}\left\{\partial_{t}: H_{1}(F, M)_{t} \rightarrow H_{1}(M)_{t}\right\}$.

In our case the radical of the intersection form has rank $2 g: \mu_{0}=2 g, \mu_{-}=1$ and $\mu_{+}=0$. If $g+1+e$ is odd, then $H_{1}(F, M)_{t}=0$, so $I=0$ and $I^{\perp}=H_{1}(M)_{t}$. For $g+1+e$ even $\bar{H}_{2}(F)$ is generated by $E-(g+1) f$, with self-intersection $-(g+1)$. In this case the isotropic subgroup $I$ has order two.

We recall the definition of smoothing data [loc. cit., 4.16] in the case $\mu_{+}=0$. Let $A$ be a finitely generated abelian group, and $q: A_{t} \rightarrow \mathbb{Q} / \mathbb{Z}$ a nonsingular quadratic function on its torsion part. Then $\mathcal{S}(A, q)$ is the set of equivalence classes of 4-tuples $(V, Q, I, i)$, where $(V, Q)$ is a negative semi definite ordinary lattice, $I$ is a $q$-isotropic subspace of $A_{t}$, and $i: V^{*} / B^{\prime}(V) \rightarrow A / I$ is an injective homomorphism with finite cokernel, which induces an isomorphism $\bar{V}^{\#} / \bar{V} \rightarrow i^{\perp} / I$ (here $B^{\prime}: V \rightarrow V^{*}$ is the adjoint of the bilinear part of $\left.Q\right)$; two 4 -tuples $\left(V_{1}, Q_{1}, I_{1}, i_{1}\right)$ and $\left(V_{2}, Q_{2}, I_{2}, i_{2}\right)$ are equivalent, if $I_{1}=I_{2}$ and there exists an isomorphism $\Phi:\left(V_{1}, Q_{1}\right) \rightarrow\left(V_{2}, Q_{2}\right)$ such that $i_{2}=\phi \circ i_{1}$, where $\phi$ is induced by $\Phi$.

Let $J$ be the preimage of $\operatorname{Im}(i)$ in $A$. Then $J \cap A_{t}=I^{\perp}$ (so $J$ determines $I)$. By [loc. cit., 4.17] the elements in $\mathcal{S}(A, q)$, which give the same triple $(V, Q, J)$ form $O\left(q_{I}\right)$-orbit, where $O\left(q_{I}\right)$ is the group of linear automorphims preserving the quadratic function $q_{I}$ on $I^{\perp} / I$. Each orbit is equivalent to a coset space in $O\left(q_{I}\right)$ of $\operatorname{Im}\left\{O(\bar{Q}) \rightarrow O\left(q_{I}\right)\right\}$.

For all our smoothings we have $H_{1}(M)_{t} / I^{\perp} \cong H_{1}(F)$. In terms of $J$ this means that $A_{t}+J=A$. In [loc. cit.] this condition is shown to hold for all minimally elliptic singularities, with the concept of permissible quotients. Denote for our singularity $X$ by $\mathcal{S}(X)$ the subset of $\mathcal{S}\left(H_{1}(M), q\right)$ of lattices with $\mu_{-}=1$, $\mu_{0}=2 g$, for which $H_{1}(M)_{t}+J=H_{1}(M)$. The set of smoothing components maps to $\mathcal{S}(X)$.

(3.14) Proposition. Suppose $g$ is even. Then the set $\mathcal{S}(X)$ has $1+2^{2 g}$ elements. On the set of even smoothing components (i.e. with $g+1+e$ even) the map to $\mathcal{S}(X)$ is injective, whereas the odd components all map to the same element.

Proof. We first do not impose restrictions on $g$. By [Looijenga-Wahl 1986, Ex. 4.6] the group $H_{1}(M)_{t}$ has an isotropic subgroup of order $r$, if $4 g+4=r^{2} s$, $2 g-2=r u$ with $s(1+r) \equiv u \quad(\bmod 2)$. So $8 \equiv 0 \quad(\bmod r)$. Possible values are $r=2, u=g-1, s=g+1$, for arbitrary $g$. If $g=8 k+3$, then also $(r, s, u)=(4,2 k+1,4 k+1)$ is a solution.

Next we determine $O\left(q_{I}\right)$. Write $g+1=h$. First consider the case $I=0$. We look for a map $\bar{e} \mapsto d \bar{e}$ such that for all $m$ :

$$
8 h\left(q(m d \bar{e})-q(m \bar{e})=(d-1)\left(m^{2}(d+1)-6 h m+4 m\right) \equiv 0 \quad(\bmod 8 h) .\right.
$$

Let $G=\operatorname{gcd}(d-1,8 h)$, and $8 h=a G$, then $m^{2}(d+1)-6 h m+4 m \equiv 0 \quad(\bmod a)$, and therefore $d+1 \equiv 6 h-4,2(d+1) \equiv 0$, so $16 \equiv 0 \quad(\bmod a)$. Because $d<4 h$, possible values for $a$ are 4,8 and 16. Only for $a=8$ we find solutions, given by $d=h+1, h=8 k-2$ or $d=3 h+1, h=8 k+2$. In these cases $O\left(q_{I}\right)$ is a group of order two. If $I$ is a group of order 2 , with $2 h \bar{e}$ as generator, then $I^{\perp} / I \cong \mathbb{Z} / h$, and we find that for $h=8 k, d=4 k+1$, the group $O\left(q_{I}\right)$ has order two. 
So if $h$ is odd, then the group $O\left(q_{I}\right)$ is trivial, and the number of elements $\mathcal{S}(X)$ is the number of triples $(V, Q, J)$. If $I=0$, then $J=H_{1}(M)_{t}$, and this is the only possibility. Now suppose that $I$ is a group of order two. In [loc. cit., 6.2] it is shown that $\operatorname{Hom}\left(H_{1}(M) / H_{1}(M)_{t}, H_{1}(M)_{t} / I^{\perp}\right)$ acts simply transitive on the collection of subgroups $J$ of $H_{1}(M)$ with $H_{1}(M)_{t} \cap J=I^{\perp}$ and $H_{1}(M)_{t}+J=H_{1}(M)$. In our case this group is isomorphic to $(\mathbb{Z} / 2)^{2 g}$.

To show injectivity, we have to identify this action with the action of the $\mathcal{B}^{+}$. The group $H_{2}(F, \partial F)$ is isomorphic to $H_{2}(S, C)$; we descibe this group with the exact sequence of the pair $(S, C)$. For $H_{2}(S)$ we have two generators, $E$ and $f$, and $C \sim 2 E$. Take a basis of $H_{1}(C)$, in the traditional way (cf. [Mumford 1984, p. $3.75]$ ): choose a certain system of $2 g$ paths in $\mathbb{P}^{1}-B, B$ the set of branch points of $\pi: C \rightarrow \mathbb{P}^{1}$, which each encircle an even number of branch points, and lift them to $C$; in $\mathbb{P}^{1}$ such a path $\alpha_{i}$ bounds a disc $D_{i}$. Now we consider the surface $S \stackrel{\pi}{\longrightarrow} \mathbb{P}^{1}$. In $S$ a lift of $\alpha_{i}$ to $C$ is the boundary of a lift $\Delta_{i}$ of $D_{i}$ to $S$. Then $E, f$ and the $\Delta_{i}$ generate $H_{2}(F, \partial F)$. The map to $H_{1}(M)$ is given by intersecting on $S$ with the boundary $M$ of a tubular neighbourhood of $C$ (with as orientation the one induced by $F$ ). The manifold $M$ is a circle bundle over $C$, with Euler number $-(4 g+4)$; let $\gamma$ be the homology class of a fibre, then $\gamma$ generates $H_{1}(M)_{t}$. A general fibre of $S \stackrel{\pi}{\longrightarrow} \mathbb{P}^{1}$ intersects $M$ in two circles; let furthermore $A_{i}$ be a lift of $\alpha_{i}$ to $M$. Then the map $\partial: H_{2}(F, \partial F) \rightarrow H_{1}(M)$ is given by $\partial(f)=2 \gamma, \partial(E)=2(g+1) \gamma$ and $\partial\left(\Delta_{i}\right)=A_{i}$.

Consider now $S^{\prime}=\operatorname{elm}_{T}(S)$, giving the Milnor fibre $F^{\prime}$. Let $\alpha_{i}$ encircle a point $P \in T$; we may suppose that $\Delta_{i}$ does not pass through $P$, but intersects the fibre $\pi^{-1}(P)$ once, outside the tubular neighbourhood of $C$.. The strict transform $\Delta_{i}^{\prime}$ on $S^{\prime}$ is the element of $H_{2}\left(F^{\prime}, \partial F^{\prime}\right)$, which lifts $\alpha_{i}$. But on $S^{\prime}$ the $\operatorname{disc} \Delta_{i}$ intersects $C^{\prime}$ transversally, and therefore intersects $M$ in a circle. So $\partial^{\prime}\left(\Delta_{i}^{\prime}\right)=A_{i}+t_{i} \gamma$, where $t_{i}$ is the number of branch points lying in $D_{i}$. If $t_{i}$ is odd, then $\partial^{\prime}\left(\Delta_{i}^{\prime}\right)$ does not lie in $\partial\left(H_{2} F, \partial F\right)$. This construction establishes an isomorphism between $\mathcal{B}^{+}$and $\operatorname{Hom}\left(H_{1}(M) / H_{1}(M)_{t}, H_{1}(M)_{t} / I^{\perp}\right)$.

(3.15) Remark. In the case $g=3$ an isotropic subgroup $I \subset H_{1}(M)_{t}$ of order 4 occurs for a smoothing of $X\left(C, K^{4}\right)$ : a non hyperelliptic curve is hyperplane section of 4 -fold Veronese embedding of $\mathbb{P}^{2}$, i.e. $\mathbb{P}^{2}$ embedded with $\mathcal{O}(4)$. It is well known that $H_{1}\left(\mathbb{P}^{2} \backslash C\right)=\pi_{1}\left(\mathbb{P}^{2} \backslash C\right)=\mathbb{Z} / 4$.

(3.16) Remark. For a simple elliptic singularity of multiplicity $8=4 g+4$ the topological computation still work; in this case for $I=0$ the group $O\left(q_{I}\right)$ is trivial. Then the permissible smoothing data form a set of $1+2^{2}=5$ elements [LooijengaWahl 1986, 6.4]. The number of smoothing components is also five; there are four 'even' components and one 'odd' component.

Let $X$ be the cone over an elliptic curve $E$ of degree 8 , embedded with the linear system $L$; so $\phi_{L}: E \rightarrow \mathbb{P}^{7}$. We identify $\operatorname{Pic}^{8}(E)$ with $\operatorname{Jac}(E) \cong E$ in such a way that $L$ represents the origin of the group law: we write $L=8 P$. There are 4 line bundles $M$ of degree 4 with $M^{\otimes 2}=L$. Each of these embeds $E$ in $\mathbb{P}^{3}$; then $\phi_{L}$ is the composition of $\phi_{M}$ with the the Veronese embedding $V: \mathbb{P}^{3} \rightarrow \mathbb{P}^{9}$. The curve $\phi_{M}(E)$ lies on a pencil of quadrics, which is transformed by $V$ in a pencil of hyperplane sections of $V\left(\mathbb{P}^{3}\right)$; the common intersection is $E$. This construction 
gives a 2-dimensional linear subspace (a cone over $\mathbb{P}^{1}$ ) in the negative part of the base space of the versal deformation of $X$. The general quadric in the pencil is non-singular and has two rulings, but there are four singular quadrics, which are cones with one ruling; the resulting double cover of $\mathbb{P}^{1}$ is isomorphic to $E$. The fifth component is isomorphic to the cone over $E$; given a point $Q \in E$, we embed $E$ in the plane with the linear system $|2 P+Q|$, then embed $\mathbb{P}^{2}$ with the triple Veronese embedding $V_{3}$ in $\mathbb{P}^{9}$, and project the surface $V_{3}\left(\mathbb{P}^{2}\right)$ from the point $V_{3} \circ \phi_{|2 P+Q|}(3 Q)$ to $\mathbb{P}^{8}$. Each of the resulting surfaces has a unique ruling.

Each ruling determines a $g_{2}^{1} \in \mathrm{Pic}^{2} \cong E$; on the even components a given $g_{2}^{1}$ occurs exactly once, and on the odd component four times. The operations $\operatorname{elm}_{T}$, with $T$ a subset of the set of branch points $\mathcal{B}$ of the $g_{2}^{1}$, permute the $2^{3}$ points in the projectivised base space.

(3.17) Other degrees. The structure of the equations (see (2.2) and (2.12)) and the dimension of $T_{X}^{2}(-2)$, which is $d-2 g-3$, two less than the number of equations, which cut out the curve on the scroll, lead us to expect that the base space $S^{-}$in negative degree is a complete intersection of dimension $4 g+5-d$, if $2 g+4 \leq d \leq 4 g+5$. The number of equations will probably increase linearly for $d>4 g+5$, until it is $(g+1)(2 g+3)$, the number of quadratic monomials in $2 g+2$ variables.

For $d=4 g+5$ the cone $X$ has only conical deformations [Tendian 1992a], and therefore all infinitesimal deformations of negative degree are obstructed. In this case we have indeed a zero-dimensional complete intersection.

For $d=4 g+3$ the space $S^{-}$should be the cone over a complete intersection curve of degree $2^{2 g}$, which is a $2^{2 g}$-fold unbranched covering of $C$. One finds this degree from the genus formula's. From the point of view of the surfaces, which correspond to one parameter deformations, we get the following picture. A surface $S$ of degree $4 g+3$ is a scroll, blown up in one point $P$ of the hyperelliptic curve $C$. The fibration $\pi: S \rightarrow \mathbb{P}^{1}$ has one singular fibre, which intersects $C$ in $P$ and in $\bar{P}$, the image of $P$ under the hyperelliptic involution. By blowing down one of the two $(-1)$-curves in the exceptional fibre we get a ruled surface with a minimal section $E_{0}$ with $E_{0}^{2}=-e$; if we blow down the other curve, then the parity of $e$ is just the opposite. Suppose that blowing down the curve through $P$ gives an even $e$. As we have seen, there are $2^{2 g}$ different surfaces with $e$ even, such that the characteristic linear system on $C$ is a given linear system $|L+P|$. Altogether we get a covering of $\mathbb{P}^{1}$ of degree $2^{2 g+1}$, with simple branching at the Weierstraß points, so indeed a $2^{2 g}$-fold unbranched covering of $C$. In particular, there is only one smoothing component, except of course when $g=3$.

We leave it to the reader to continue this description for still lower values of the degree $d$. 


\section{Bibliography}

EGA IV A. Grothendieck, Éléments de géométrie algébrique. Rédigés avec la collaboration de J. Dieudonné. IV, Étude locale des schémas et des morphismes de schémas. Publ. Math. IHES 20 (1964), 24 (1965), 28 (1966), 32 (1967).

Enrico Arbarello e Maurizio Cornalba, Su una congettura di Petri. Comment. Math. Helv. 56, (1981), 1-38.

Enrico Arbarello and Eduardo Sernesi, Petri's Approach to the Study of the Ideal Associated to a Special Divisor. Invent. Math. 49, (1978), 99-119.

Dave Bayer and Mike Stillman, Macaulay: A system for computation in algebraic geometry and commutative algebra. Source and object code available for Unix and Macintosh computers. Contact the authors, or download from zariski.harvard.edu via anonymous ftp.

Kurt Behnke and Jan Arthur Christophersen, Hypersurface sections and obstructions (rational surface singularities). Comp. Math. 77 (1991), 233-268.

Guido Castelnuovo, Sulle superficie algebriche le cui sezioni piane sono curve iperellitiche. Rend. Circ. Mat. Palermo 4 (1890), 73-88.

Ciro Ciliberto, Joe Harris and Rick Miranda On the surjectivity of the Wahl map. Duke J. Math. 57 (1988), 829-858.

Fabio Conforto, Le superficie razionale. Bologna, Zanichelli, 1939.

R. Drewes, Infinitesimale Deformationen von Kegeln über transkanonisch eingebetteten hyperelliptischen Kurven. Abh. Math. Sem. Univ. Hamburg 59 (1989), 269-280.

Mark L. Green, Koszul cohomology and the geometry of projective varieties. J. Diff. Geom. 19 (1984), 125-171.

Gert-Martin Greuel, On deformations of curves and a formula of Deligne. In: Algebraic Geometry, La Rábida 1981, pp. 141-168. Berlin etc., Springer 1982 (Lect. Notes in Math.; 961).

Robin Hartshorne, Algebraic Geometry. Berlin etc., Springer 1977.

Steven L. Kleiman, The Enumerative Theory of Singularities. In: Real and Complex Singularities, Oslo 1976, pp. 297-396 Alphen a/d Rijn, Sijthoff \& Noordhoff 1977.

Paulette Lévy-Bruhl-Mathieu. Étude des surfaces d'ordre $p-2+h(0 \leq h<p)$ passant par une courbe canonique de genre $p$. Application à la classification des courbes alǵebriques de genre inférieur à 11. C. R. Ac. Sc. Paris, 236 (1953), 2032- 2034.

Eduard Looijenga, The smoothing components of a triangle singularity II. Math. Ann. 269 (1984), 357-387.

Eduard Looijenga and Jonathan Wahl, Quadratic functions and smoothing surface singularities. Topology 25 (1986), 261-291.

David Mumford, A remark on the paper of M. Schlessinger. Rice Univ. Studies 59 (1973), 113-117.

David Mumford, Tata Lectures on Theta II. Boston etc., Birkhäuser 1984 (Progress in Math.; 28). 
Ragni Piene, Polar classes of singular varieties. Ann. Sc. Éc. Norm. Sup. 11 (1978) $247-276$.

Henry C. Pinkham, Deformations of algebraic varieties with $G_{m}$-action. Astérisque 20 (1970).

Miles Reid, Surfaces with $p_{g}=3, K^{2}=4$ according to E. Horikawa and D. Dicks. Text of a lecture, Univ. of Utah and Univ. of Tokyo 1989.

Michael Schlessinger, On rigid singularities. Rice Univ. Studies 59 (1973), 147-162.

Frank-Olaf Schreyer, Syzygies of Canonical Curves and Special Linear Series. Math. Ann. 275 (1986), 105-137.

Francesco Severi, Vorlesungen über Algebraische Geometrie. Leipzig, Berlin, B. G. Teubner, 1921.

Jan Stevens, On the number of points determining a canonical curve. Indag. Math. 51 (1989), 485-494.

Jan Stevens, On deformations of singularities of low dimension and codimension: base spaces and smoothing components. Preprint 1991 (Schriftenreihe Forschungsschwerpunkt Komplexe Mannigfaltigkeiten; 129).

Sonny Tendian, Deformations of Cones and the Gaussian-Wahl Map. Preprint 1990.

Sonny Tendian, Surfaces of degree $d$ with sectional genus $g$ in $\mathbb{P}^{d+1-g}$ and deformations of cones. Duke J. Math. 65 (1992), 157-185.

Sonny Tendian, Extensions of hyperelliptic curves. Preprint 1992.

Jonathan M. Wahl, The Jacobian algebra of a graded Gorenstein Singularity. Duke J. Math. 55 (1987) 843-871.

Jonathan Wahl, Deformations of Quasi-homogeneous Surface Singularities. Math. Ann. 280 (1988), 105-128.

Jonathan Wahl, Introduction to Gaussian maps on an algebraic curve. Notes prepared in connection with lectures at the Trieste Conference on Projective Varieties, June 1989.

Jonathan Wahl, Gaussian maps on algebraic curves. J. Diff Geom. 32 (1990), $77-98$.

Address of the author:

Mathematisches Seminar der Universität Hamburg

Bundesstraße 55, D 2000 Hamburg 13, Germany

E-mail: (new) stevens@geomat.math.uni-hamburg.de

(old) ms00010@dhhuni4.bitnet 